\title{
Paramagnetic spin labeling of a bacterial DnaB helicase for solid- state NMR
}

\section{Journal Article}

Author(s):

Zehnder, Johannes; Cadalbert, Riccardo; Yulikov, Maxim; Künze, Georg; Wiegand, Thomas

Publication date:

2021-11

Permanent link:

https://doi.org/10.3929/ethz-b-000508272

\section{Rights / license:}

Creative Commons Attribution-NonCommercial-NoDerivatives 4.0 International

Originally published in:

Journal of Magnetic Resonance 332, https://doi.org/10.1016/j.jmr.2021.107075 


\title{
Paramagnetic spin labeling of a bacterial DnaB helicase for solid-state NMR
}

\author{
Johannes Zehnder ${ }^{\mathrm{a}}$, Riccardo Cadalbert ${ }^{\mathrm{a}}$, Maxim Yulikov ${ }^{\mathrm{a}}$, Georg Künze ${ }^{\mathrm{b}, *}$, Thomas Wiegand ${ }^{\mathrm{a}, \mathrm{c}, \mathrm{d}, *}$ \\ ${ }^{a}$ Physical Chemistry, ETH Zurich, 8093 Zurich, Switzerland \\ ${ }^{\mathrm{b}}$ Institute for Drug Discovery, Medical School, Leipzig University, 04103 Leipzig, Germany \\ ${ }^{\mathrm{c}}$ Max-Planck-Institute for Chemical Energy Conversion, Stiftstr. 34-36, 45470 Mülheim an der Ruhr, Germany \\ ${ }^{\mathrm{d}}$ Institute of Technical and Macromolecular Chemistry, RWTH Aachen University, Worringerweg 1, 52074 Aachen, Germany
}

\section{A R T I C L E I N F O}

\section{Article history:}

Received 19 July 2021

Revised 16 September 2021

Accepted 17 September 2021

Available online 21 September 2021

\section{Keywords:}

Solid-state NMR

Spin labeling

Paramagnetic Relaxation Enhancement

Molecular modeling

Motor protein

\begin{abstract}
A B S T R A C T
Labeling of biomolecules with a paramagnetic probe for nuclear magnetic resonance (NMR) spectroscopy enables determining long-range distance restraints, which are otherwise not accessible by classically used dipolar coupling-based NMR approaches. Distance restraints derived from paramagnetic relaxation enhancements (PREs) can facilitate the structure determination of large proteins and protein complexes. We herein present the site-directed labeling of the large oligomeric bacterial DnaB helicase from Helicobacter pylori with cysteine-reactive maleimide tags carrying either a nitroxide radical or a lanthanide ion. The success of the labeling reaction was followed by quantitative continuous-wave electron paramagnetic resonance (EPR) experiments performed on the nitroxide-labeled protein. PREs were extracted site-specifically from 2D and 3D solid-state NMR spectra. A good agreement with predicted PRE values, derived by computational modeling of nitroxide and $\mathrm{Gd}^{3+}$ tags in the low-resolution DnaB crystal structure, was found. Comparison of experimental PREs and model-predicted spin label-nucleus distances indicated that the size of the "blind sphere" around the paramagnetic center, in which NMR resonances are not detected, is slightly larger for $\mathrm{Gd}^{3+}(\sim 14 \AA)$ than for nitroxide $(\sim 11 \AA)$ in ${ }^{13} \mathrm{C}$-detected 2D spectra of DnaB. We also present $\mathrm{Gd}^{3+}-\mathrm{Gd}^{3+}$ dipolar electron-electron resonance EPR experiments on DnaB supporting the conclusion that DnaB was present as a hexameric assembly.
\end{abstract}

(c) 2021 The Author(s). Published by Elsevier Inc. This is an open access article under the CC BY-NC-ND license (http://creativecommons.org/licenses/by-nc-nd/4.0/).

\section{Introduction}

Solid-state NMR spectroscopy represents a versatile tool for the structural characterization of proteins as well as for probing their conformational dynamics [1,2]. Technical advances in solid-state NMR spectroscopy, such as the availability of high magnetic-field strengths $[3,4]$, high rotation frequencies in magic-angle spinning (MAS) experiments [5,6] as well as the development of highly efficient multidimensional radiofrequency pulse sequences, have enabled the routine assignment of uniformly ${ }^{13} \mathrm{C}-{ }^{15} \mathrm{~N}$ labeled proteins with molecular weights up to $\sim 30 \mathrm{kDa}$ [7], and determination of the structures of small to medium-sized proteins $(<100-150$ amino acids per symmetric monomer). Structure determination mostly relies on the collection of a large number of distance restraints extracted from dipolar coupling-based NMR

\footnotetext{
* Corresponding authors at: Physical Chemistry, ETH Zurich, 8093 Zurich, Switzerland (T. Wiegand) and Institute for Drug Discovery, Leipzig University, 04103 Leipzig, Germany (G. Künze).

E-mail addresses: georg.kuenze@uni-leipzig.de (G. Künze), thomas.wiegand@_ phys.chem.ethz.ch (T. Wiegand).
}

experiments and backbone torsion-angle restraints derived from the chemical shifts, e.g., via TALOS predictions [8]. Since the dipolar coupling constant between two nuclei (denoted here by $i$ and $j$ ), $D_{i j}$, is inversely proportional to the third power of the distance separating the two nuclei, the detected spin pairs have short distances. Collection of long-range restraints (with $|\mathrm{i}-\mathrm{j}|>4$ ), with small $D_{i j}$ values, requires recording spectra with long mixing times. While for large proteins signal overlap in the spectra recorded at long mixing times can become problematic, the resolution can be improved in these cases by going to higher dimensions or by increasing the external magnetic field strength $[3,4]$.

Pseudo contact shifts (PCSs) or paramagnetic relaxation enhancements (PREs), which are caused by the interaction of nuclear spins with metal ions or radicals bearing unpaired electrons $[9,10]$, are a promising alternative to dipolar couplingbased distance restraints. Due to the much larger gyromagnetic ratio of electrons, PCSs and PREs can be observed over long distances ( 20-25 ̊, for some lanthanides even up to $40 \AA$ [11]) providing long-range structural restraints. By contrast, typical dipolar nuclear distance restraints do not exceed $8 \AA$. 
The relaxation properties of nuclei in a protein containing a paramagnetic center are affected by PREs [9,12-14]. This relaxation phenomenon is treated within the Solomon-Bloembergen relaxation theory $[15,16]$ and originates from the modulation of the dipolar part of the electron-nucleus hyperfine coupling [15]. The mathematical expressions for the contribution of the PRE to the longitudinal $\left(\Gamma_{1}\right)$ and transverse $\left(\Gamma_{2}\right)$ relaxation rate constants in the solid state, respectively, are given by Eqs. (1) and (2):

$\Gamma_{1}=\frac{2}{15}\left(\frac{\mu_{0}}{4 \pi}\right)^{2} \frac{\gamma_{n}^{2} g_{e}^{2} \beta^{2} S(S+1)}{r^{6}}\left(\frac{3 T_{1 e}}{1+\omega_{n}^{2} T_{1 e}^{2}}+\frac{7 T_{2 e}}{1+\omega_{e}^{2} T_{2 e}^{2}}\right)$

$$
\begin{aligned}
\Gamma_{1 \rho} \approx & \Gamma_{2} \\
= & \frac{1}{15}\left(\frac{\mu_{0}}{4 \pi}\right)^{2} \\
& \times \frac{\gamma_{n}^{2} g_{e}^{2} \beta^{2} S(S+1)}{r^{6}}\left(4 T_{1 e}+\frac{3 T_{1 e}}{1+\omega_{n}^{2} T_{1 e}^{2}}+\frac{13 T_{2 e}}{1+\omega_{e}^{2} T_{2 e}^{2}}\right)
\end{aligned}
$$

Herein, $\omega_{\mathrm{n}}$ and $\omega_{\mathrm{e}}$ represent the nuclear and electron Larmor frequencies. $T_{1 e}$ denotes the longitudinal electron spin relaxation time constant with which the correlation time is approximated (with $T_{1 \mathrm{e}} \approx T_{2 \mathrm{e}}$ )[17,18] neglecting chemical-exchange effects.

Different sample preparation schemes for paramagnetic NMR studies have been described. In case of metalloproteins, naturally occurring metal binding sites can be used and loaded with transition metals or lanthanide ions [19]. For instance, the diamagnetic $\mathrm{Mg}^{2+}$ cofactor in ATP-driven proteins can be exchanged with paramagnetic metal ions [20,21]. Additionally, diamagnetic proteins and nucleic acids [22] can be modified at specific positions with covalently bound paramagnetic tags [12,23], e.g., by using tags that react with the thiol groups of free cysteines which can be sitespecifically introduced into proteins by mutagenesis [24-27]. In case of membrane proteins, transition metal ions can be attached to the phospholipid head groups of the membrane by chelating tags [28-30]. Another alternative is to engineer completely new lanthanide binding sites into proteins [31-33].

PCSs and PREs can be integrated in both, resonance assignment and structure determination. The relative strengths of the two effects depend on the chosen paramagnetic tag or metal ion [10]. PREs and PCSs have been employed to support the 3D assignment of uniformly or amino acid selectively labeled proteins by comparing experimental PRE and PCS data with values calculated from the 3D structure, if the latter is known [34-40]. PCSs have been applied in various solid-state NMR studies [41-45] and PREs have been employed, for instance, in the structure determination of GB1 [46-48].

We herein expand on these previous studies by applying paramagnetic tagging and solid-state NMR to the large bacterial DnaB helicase from Helicobacter pylori [49,50]. DnaB is an ATP-fueled motor protein that unwinds double-stranded DNA into single strands during DNA replication [51]. In its active conformation, DnaB forms a ring-shaped hexamer with each polypeptide chain comprising 488 amino acid residues. The spin labeling of DnaB using a nitroxide (3-maleimido-PROXYL) and a lanthanide tag $\left(\mathrm{Gd}^{3+}\right.$-maleimido-DOTA), respectively, is described. The efficiency of the labeling reaction was monitored by quantitative EPR spectroscopy carried out on the nitroxide-labeled protein. Sitespecific PREs were extracted as signal attenuations from 2D ${ }^{13} \mathrm{C}-{ }^{13} \mathrm{C}$ Dipolar Assisted Rotational Resonance (DARR) [52,53] and 3D NCACB correlation spectra using dia- and paramagnetic protein samples, for the latter without dilution with diamagnetic protein [42]. The PREs were compared to theoretical values. Those were obtained by generating ensemble distribution models of the nitroxide as well as the $\mathrm{Gd}^{3+}$ tag attached to the low-resolution X-ray structure of DnaB [54] by computational modeling with
Rosetta [55]. Based on the structural models and their comparison with the experimental PRE data, we determined the blind sphere radii (i.e., the region in which NMR resonances are broadened beyond detection) for both tags. Finally, we present $\mathrm{Gd}^{3+}-\mathrm{Gd}^{3+}$ EPR distance measurements confirming that DnaB formed indeed a dimer of hexamers. Our study paves the way for combining paramagnetic solid-state NMR and EPR to unravel the interactions of DnaB with other proteins involved in DNA replication, such as the primase DnaG [56].

\section{Results and discussion}

2.1. Spin labeling of the DnaB helicase with nitroxide and lanthanide tags

Spin labeling of DnaB was achieved by covalently modifying the two native cysteine residues in DnaB (C60 and C271) with either a nitroxide tag (3-maleimido-proxyl, abbreviated with PROXYL-M in the following) or a lanthanide-chelating tag (maleimido-DOTA, abbreviated with DOTA-M in the following) (Scheme 1, vide infra). The two tags were chosen in order to compare and contrast their applicability for protein solid-state NMR studies. The diamagnetic reference for the PROXYL-M-tagged protein was obtained by reducing PROXYL-M with ascorbic acid after tagging of DnaB [22]. In case of the DOTA-M tag, the paramagnetic protein sample was prepared by using $\mathrm{Gd}^{3+}$, whereas the diamagnetic reference state was obtained by using $\mathrm{Lu}^{3+}$.

The crystal structure of $H$. pylori DnaB (resolution $6.7 \AA$ ) shows a stack-twisted double hexamer [54]. Each of the two hexamers can be described as a trimer of dimers which are assembled into a circular topology (see Fig. 1a). The N-terminal domains (NTDs) and Cterminal ATPase domains (CTDs) of each DnaB monomer contribute to two separate rings, termed NTD- and CTD-rings, that have two different symmetries and give rise to a two-tiered hexamer (Fig. 1b). While the NTD-ring is C3 symmetric and composed of a planar trimer of NTD dimers, the CTD-ring adopts a pseudo sixfold symmetry.

DnaB exhibits two cysteine residues per monomer which were employed for site-directed spin labeling. C60 is located in the loop following helix $\alpha 3$ in the NTD ring (Fig. 1c), and C271 is located on helix $\alpha 13$ in the CTD ring (Fig. 1d). Within the NTD homodimer, the two C60 residues experience different structural environments, one cysteine is oriented towards a neighboring DnaB subunit while the other cysteine is located on the outer surface of the NTD ring and is not involved in inter-subunit interactions. By contrast, within the CTD homodimer, the two copies of C271 have similar locations on the outer collar of the CTD-ring.

All four cysteine residues per DnaB homodimer are readily accessible to solvent. Within the DnaB crystal structure, the molecular surface area of the side-chain of these cysteine residues (determined using their atoms' van der Waals radii) ranges from $87 \%$ to $95 \%$ relative to the surface area of the side-chain of the free amino acid. Thus, we expected nearly stoichiometric labeling efficiency of these sites when reacted with PROXYL-M and $\mathrm{Gd}^{3+}$ DOTA-M tags.

\subsection{Quantification of spin labeling efficiency by CW-EPR spectroscopy}

NMR studies require nearly quantitative labeling efficiencies to avoid any doubling of peaks owing to the presence of the resonances of both dia- and paramagnetic protein species in the NMR spectrum. This issue is of particular importance for large proteins because of the limited resolution of their NMR spectra. The success of the labeling reaction with the PROXYL-M tag was monitored by comparing the continuous wave (CW)-EPR spectra of unbound 
a

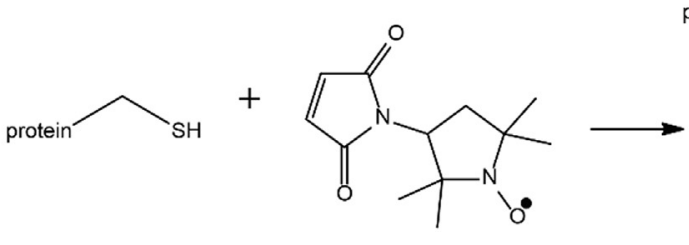

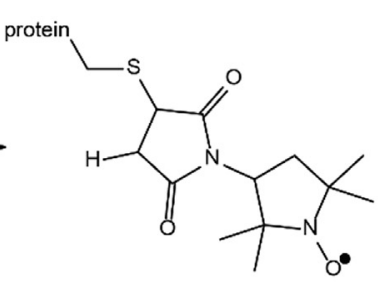

b

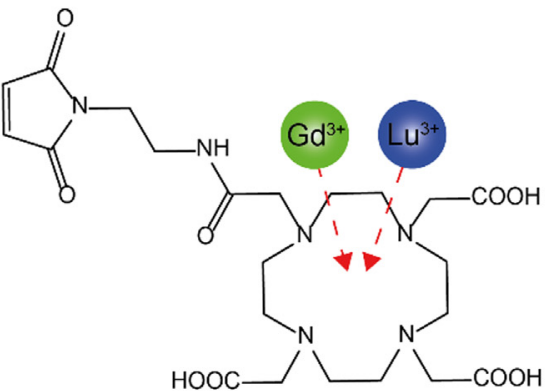

Scheme 1. 3-maleimido-proxyl (PROXYL-M) (a) and maleimido-DOTA (DOTA-M) (b) tags used for site-directed spin labeling of DnaB.

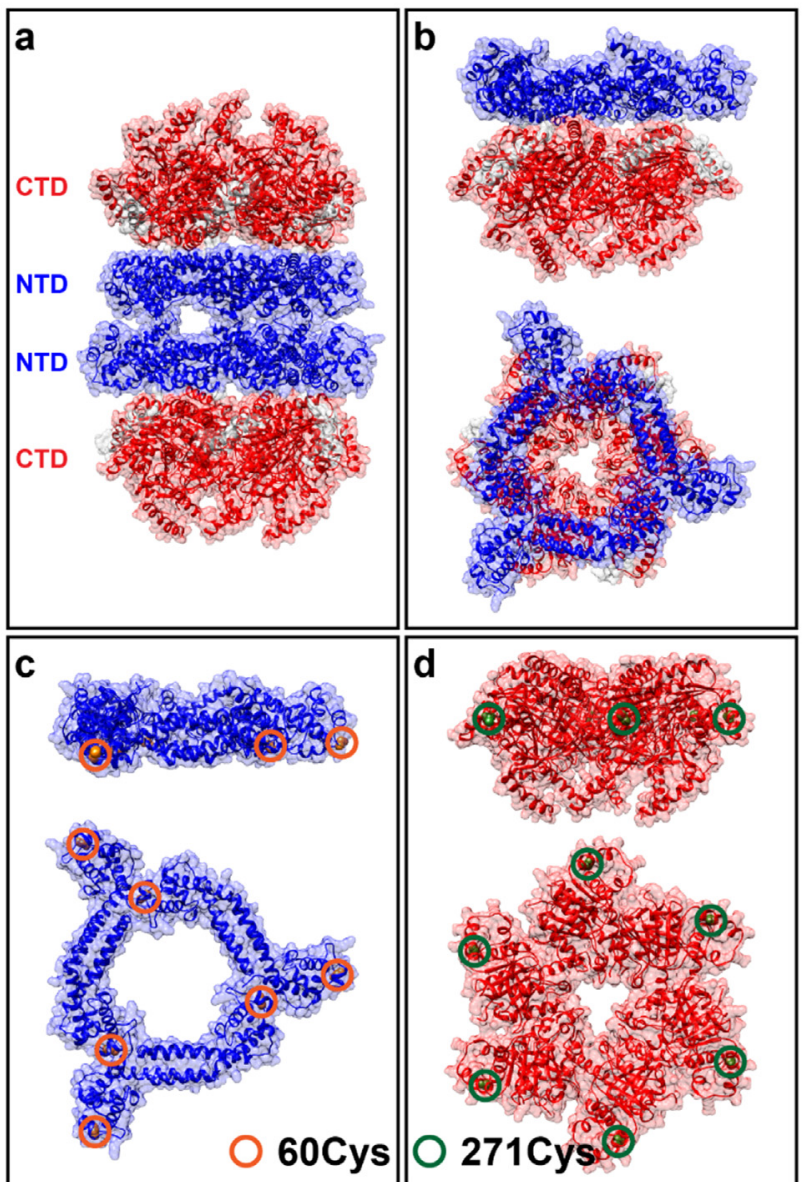

Fig. 1. Spin labeling positions in DnaB. Dodecameric assembly of DnaB with the CTD colored in red and the NTD shown in blue (a), side and top view of one DnaB hexamer (b), side and top view of the NTDs within one hexamer with C60 highlighted by red circles (c) and side and top view of the CTDs within one hexamer with C271 highlighted by green circles. PDB ID 4ZC0 has been used to visualize the protein structure. (For interpretation of the references to color in this figure legend, the reader is referred to the web version of this article.)

PROXYL-M dissolved in NMR buffer and of PROXYL-M-tagged DnaB solubilized in the same buffer (Fig. 2a and b). As expected, the EPR resonance of PROXYL-M-tagged DnaB shows a significant anisotropic broadening pointing to slow molecule tumbling and thus an immobilized nitroxide species. Double integration of the EPR spectrum allowed determining the nitroxide spin concentration and calculation of the spin labeling efficiency of DnaB of $93 \pm 20 \%$ on average for the two labeling sites per DnaB monomer. Thus, we conclude that our spin labeling protocol is suitable to produce a nearly complete tagging of DnaB with PROXYL-M, which is required for solid-state NMR studies. Additionally, we assume from this result and from the accessibility of the cysteines that the labeling efficiency for the chemically similar DOTA-M tag will be in the same range as for PROXYL-M. The quantification of the $\mathrm{Gd}^{3+}$ spin concentration from CW EPR spectra is, however, challenging due to broad resonance lines owing to large zero field splittings.

\subsection{Solid-state NMR spectra of spin labeled DnaB}

Fig. 3 shows spectral fingerprints of the ${ }^{13} \mathrm{C}-{ }^{13} \mathrm{C}$ Dipolar Assisted Rotational Resonance (DARR) spectra recorded on DnaB tagged with PROXYL-M (red) and DnaB with the reduced tag (blue). Note that the paramagnetic sample was not diluted with diamagnetic protein. This is typically done for protein preparations in the microcrystalline state to suppress intermolecular paramagnetic effects [42]. However, we assumed that the impact of such intermolecular paramagnetic effects on the NMR spectrum is negligible for a sedimented protein sample, because in this case the protein molecules have no preferred orientation relative to each other. This hypothesis is supported by the lack of long-range order described in a recent study on nucleosome sediments as shown by small-angle X-ray scattering (SAXS) [57], and also for DnaB sediments no crystalline order was detected by X-ray diffraction [58]. Possibly, the packing of DnaB in the sediment might also be less dense than in small microcrystalline proteins, such as GB1, for which paramagnetic solid-state NMR studies using diamagnetic dilution were reported [47]. However, structural details about DnaB sediments are still missing until today and more thorough investigations of such states are needed. Dilution of the paramagnetic protein with non-labeled diamagnetic protein has also practical limitations as this would further reduce the spectral signal-to-noise ratios precluding investigations of large proteins, such as DnaB.

The absence of several resonances in the spectrum of PROXYL$\mathrm{M}$-tagged DnaB, e.g., of F66 and I67, is attributed to pronounced transverse PREs experienced by these residues. This can also be seen in the 1D traces along F2 (Fig. 3) in which intensity reductions of additional peaks become apparent. Additional sections of the 2D DARR spectra showing the complete aliphatic region and another spectral fingerprint region, respectively, are displayed in Figures S1 and S2 in the supporting material. PREs were determined by comparing the intensities (peak heights) of the resonances in the spectrum of DnaB with the reduced PROXYL-M tag (dia) with those in the spectrum of PROXYL-M-tagged DnaB (para); in the following expressed as the ratio $\mathrm{I}_{\text {para }} / \mathrm{I}_{\text {dia }}$ (note that the intensities were normalized to the correlation of D39).

Similarly, solid-state NMR spectra of DOTA-M-tagged DnaB were recorded. Spectral fingerprints are given in Fig. 4. For the spectra showing the complete aliphatic region and another fingerprint region, respectively, see Figures S2 and S3. The 

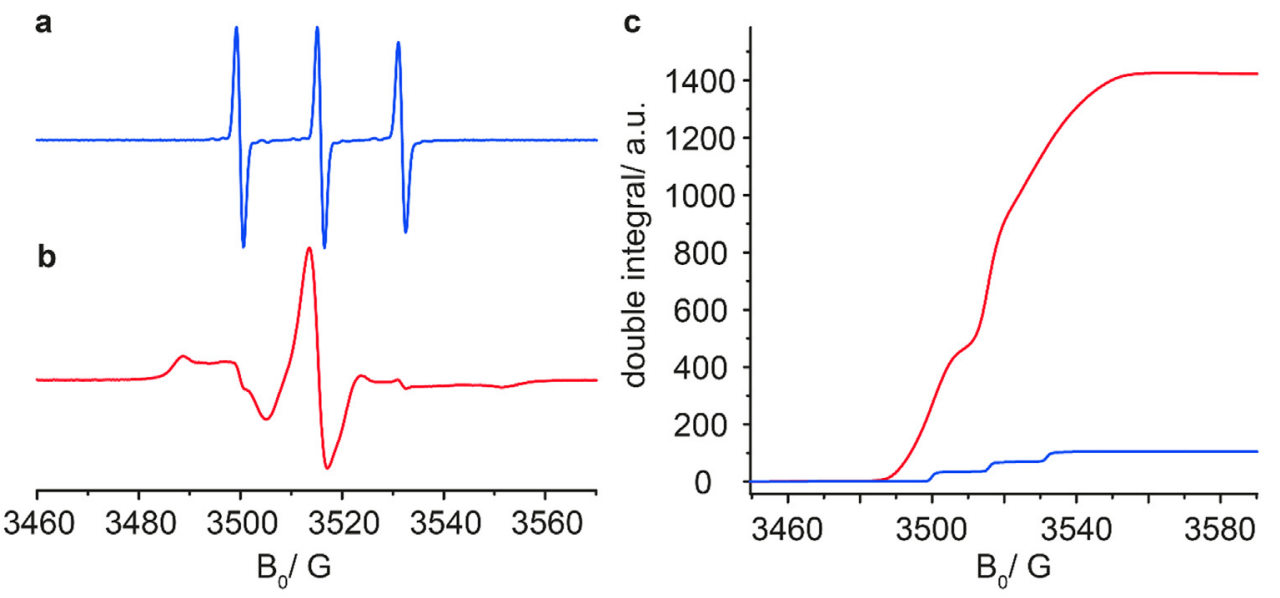

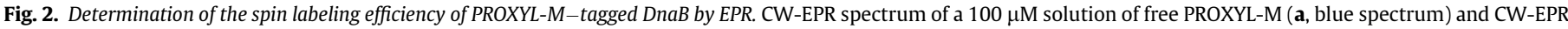

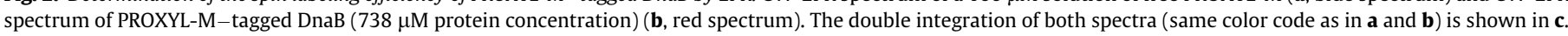

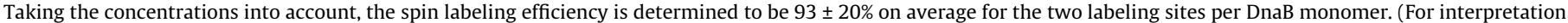
of the references to color in this figure legend, the reader is referred to the web version of this article.)
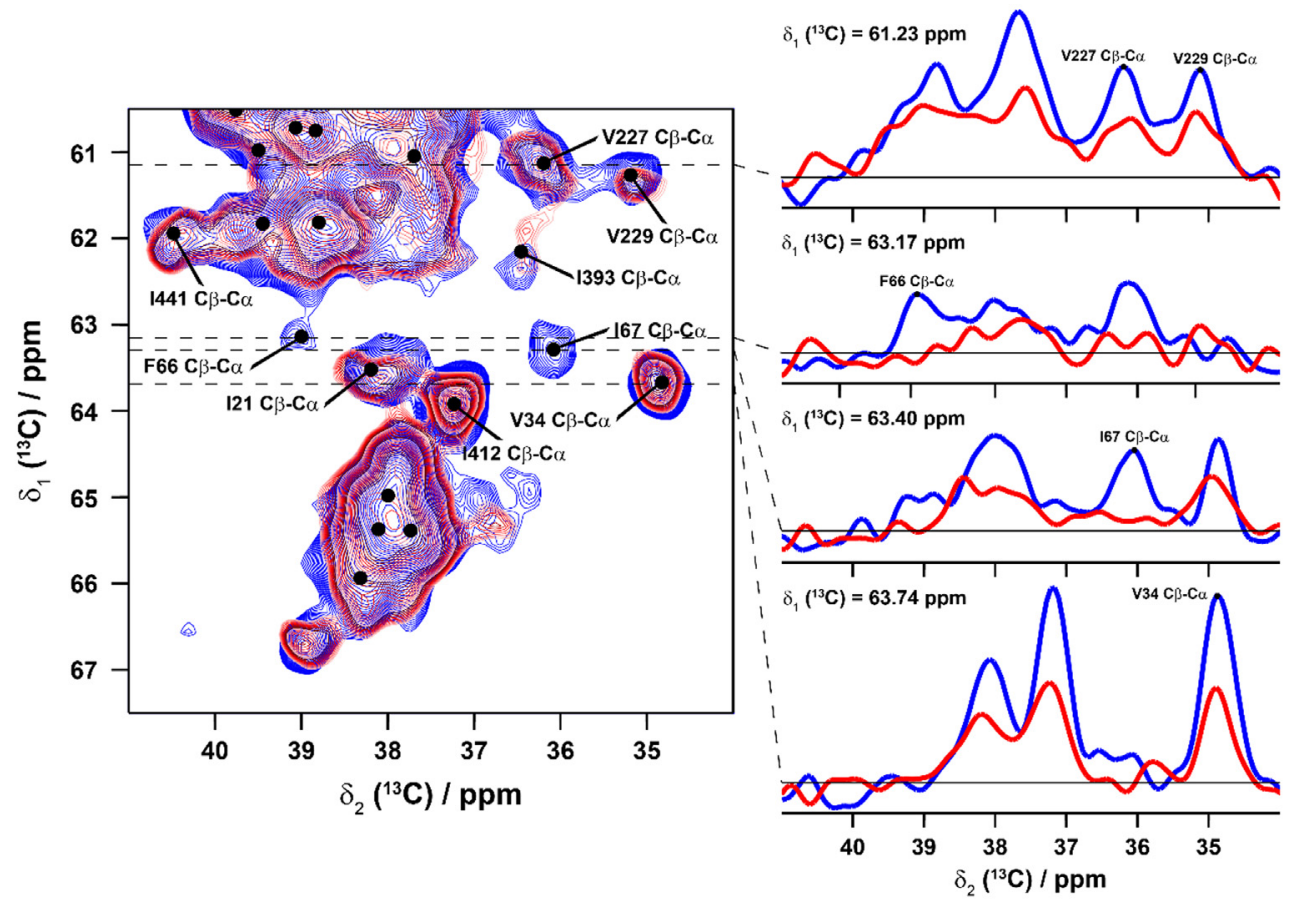

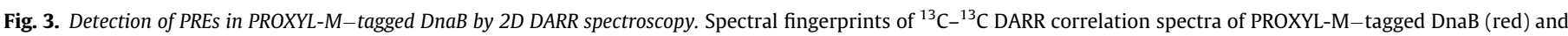

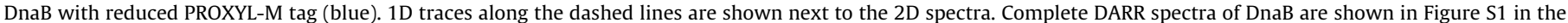
supporting material. (For interpretation of the references to color in this figure legend, the reader is referred to the web version of this article.)

spectrum of DOTA-M-tagged DnaB loaded with diamagnetic $\mathrm{Lu}^{3+}$ (reference sample) is shown in gray whereas the spectrum of DnaB tagged with paramagnetic $\mathrm{Gd}^{3+}$-DOTA-M is colored in orange. As for the PROXYL-M-tagged DnaB sample, pronounced PREs were observed in the spectrum of $\mathrm{Gd}^{3+}$-DOTA-M as cancellation of several NMR signals suggesting that labeling with $\mathrm{Gd}^{3+}$ was also nearly complete.

\subsection{Location of site-specific PREs in spin labeled DnaB and comparison with the DnaB crystal structure}

For PROXYL-M-tagged DnaB, strong relaxation enhancements $\left(\mathrm{I}_{\text {para }} / \mathrm{I}_{\text {dia }} \leq 0.25\right)$ of the NMR signals of D59, C60, P61, I62, F66,
I67, T248, and C271 were observed. Residues D59-I62 are direct neighbors of 660 within the same polypeptide chain of DnaB. T248 on helix $\alpha 11$ is spatially close to C 271 in the DnaB crystal structure model, which was initially derived from a lowresolution electron density (resolution $6.7 \AA$ ) [54]. Similarly, for $\mathrm{Gd}^{3+}$-DOTA-M-tagged DnaB, residues with pronounced PREs ( $\mathrm{I}_{\text {para }} /$ $\mathrm{I}_{\mathrm{dia}} \leq 0.25$ ) included, besides $\mathrm{C} 60$ and $\mathrm{C} 271$, those residues which are in close proximity to the spin labeling sites in the DnaB crystal structure, namely D59, P61, I62, F66, I67, and T248.

In order to more quantitatively compare our experimental PRE data with the crystal structure model of DnaB, we used a computational modeling approach. We created ensemble conformation models [59] for PROXYL-M and $\mathrm{Gd}^{3+}$-DOTA-M and determined 


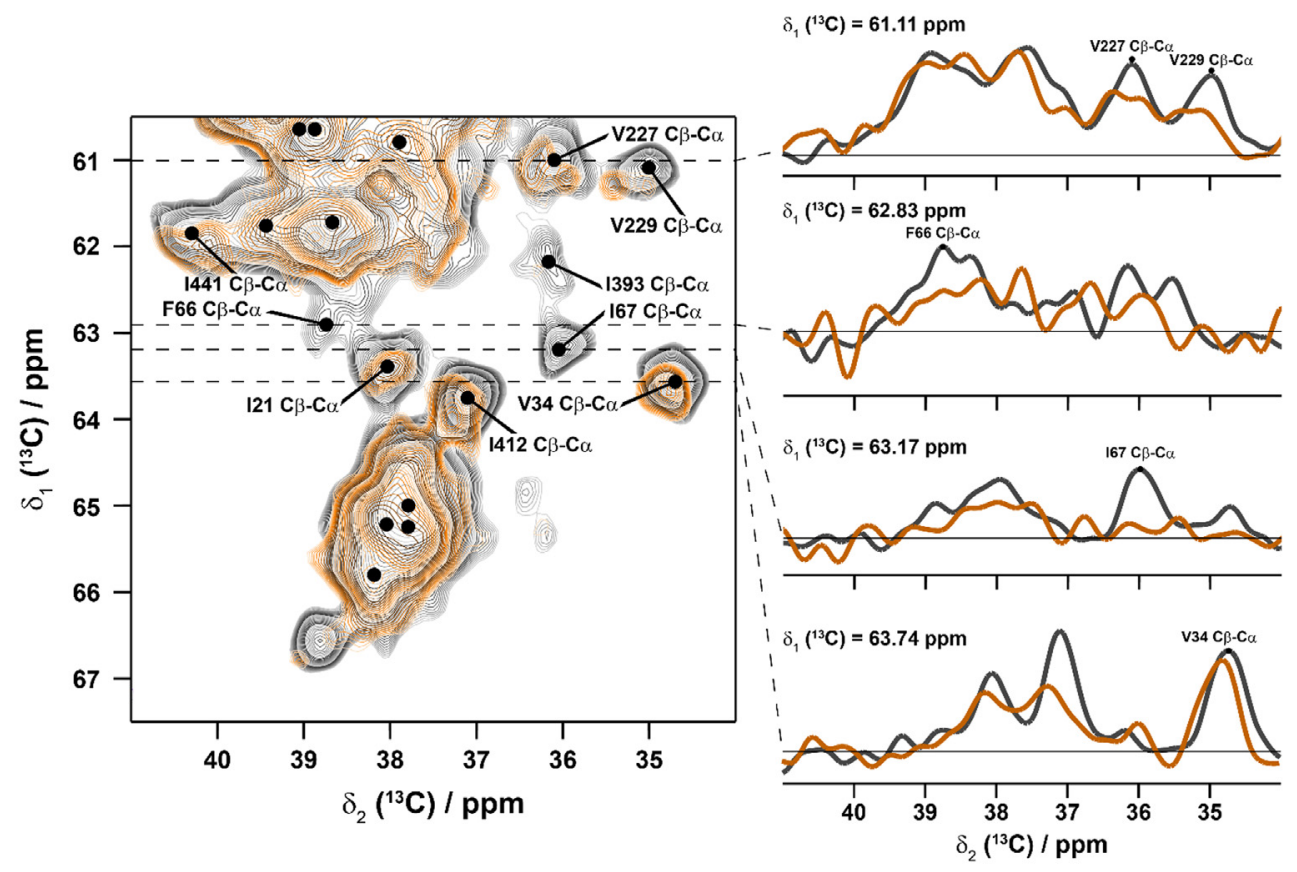

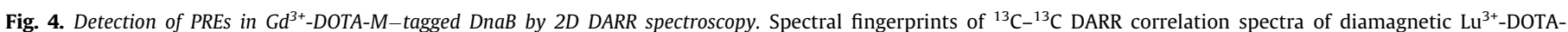

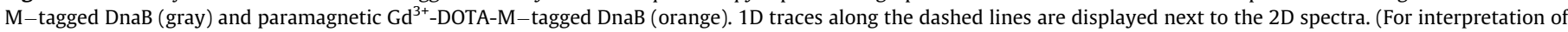
the references to color in this figure legend, the reader is referred to the web version of this article.)

effective distances between the spin labels and every amino acid residue in DnaB. First, we generated libraries of conformations for PROXYL-M and $\mathrm{Gd}^{3+}$-DOTA-M tags linked to the side-chain of Cys by sampling rotamers of small molecule fragments that matched regions in PROXYL-M-Cys or $\mathrm{Gd}^{3+}$-DOTA-M-Cys from the Crystallography Open Database [60,61] (COD) (Figure S4). Based on the resulting conformer libraries, we then modeled the conformational ensembles of PROXYL-M and $\mathrm{Gd}^{3+}$-DOTA-M spin labels at every cysteine in the DnaB dodecamer using the Rosetta program [55] as basis for distance measurements.

Fig. 5a shows the positions of the nitroxide 01 atom obtained from the distributed conformer models of PROXYL-M. Fig. 5b displays the resulting 01 atom density at each of the four spin label sites within one DnaB homodimer. We determined effective spin label-amino acid residue distances by first calculating the 01 atom center of mass at every spin label site in the DnaB dodecamer and second measuring all pairwise distances between the centers of mass and the $\mathrm{C} \alpha$ or side-chain carbon atoms of residues in the asymmetric DnaB homodimer (see Materials and Methods). Fig. $5 c$ and $5 d$ compare the experimentally observed PRE data for PROXYL-M-tagged DnaB with expected PREs that were calculated based on the effective spin label-amino acid distances. Overall, we found a favorable correlation between experimental and calculated PREs; for $82 \%$ of PREs the difference between experimental and predicted value was smaller or equal \pm 0.2 . This supports the notion that our computational models of the ensemble of PROXYL-M spin labels based on the low-resolution DnaB crystal structure provide a faithful atomistic interpretation of the experimental NMR measurements. Note that our analysis takes intramolecular PREs between the two hexamers of the dodecameric assembly into account that certainly have an impact on the measured PREs, while we have no evidences for significant intermolecular PREs (between different dodecamers, see above).

While the determination of PREs in 2D spectra of DnaB is difficult due to the large protein size, we have recently described for DnaB that this problem can, in principle, be mitigated by increasing the number of spectral dimensions [20]. Thus, we also recorded 3D NCACB spectra of PROXYL-M-tagged DnaB and apo DnaB, respectively. Figure S5a displays experimental site-specific PRE data (black data points) and simulated PRE data (red curve) for the 3D NCACB experiment. While the number of site-specifically assigned PRE data is increased in the 3D NCACB spectrum compared to the 2D DARR spectrum, the agreement between experimental and calculated PREs is not as good as for the DARR experiment (for only $56 \%$ of PREs the difference was within a margin of \pm 0.2 ). This could be attributed to the lower signal-to-noise ratios of the 3D spectra leading to larger errors of the experimental PRE values and/or to the high sensitivity of the DREAM transfer step for the chosen experimental conditions. Therefore, we interpreted those PREs only qualitatively in previous studies [21].

Fig. $6 a$ and 6 b show the positions of the $\mathrm{Gd}^{3+}$ ion of $\mathrm{Gd}^{3+}$-DOTA$\mathrm{M}$ in the DnaB dodecamer and the $\mathrm{Gd}^{3+}$ density at four different spin label sites in one DnaB homodimer, respectively. While the overall shape of the $\mathrm{Gd}^{3+}$ density distribution is similar to that one observed for the nitroxide radical, the space sampled by the $\mathrm{Gd}^{3+}$ ion in the computational simulation is normally larger compared to the nitroxide radical. This is likely a consequence of the increased number of side-chain degrees of freedom of $\mathrm{Gd}^{3+}$ DOTA-M (seven dihedral angles) versus PROXYL-M (four dihedral angles). Interestingly, the spaces occupied by PROXYL-M and $\mathrm{Gd}^{3+}$-DOTA-M at one of the two $\mathrm{C} 60$ residues in the DnaB homodimer (labeled C60-C in Fig. 5b and Fig. 6b) are slightly displaced relative to each other. This could be explained by the fact that C60 is located at the interface between two DnaB NTD dimers, which limits the conformational space for the larger $\mathrm{Gd}^{3+}$-DOTA$\mathrm{M}$ tag but leaves more room for the smaller PROXYL-M tag.

Fig. $6 c$ and d compare experimental PREs obtained on $\mathrm{Gd}^{3+}$ DOTA-M-tagged DnaB with PREs calculated from the DnaB structure by determining effective $\mathrm{Gd}^{3+}$-amino acid residue distances. Overall, structural modeling supports the experimental PRE measurements; for $63 \%$ of PREs, the agreement between experimental and calculated values is equal or better \pm 0.2 . Noteworthy, $\mathrm{I}_{\mathrm{para}} / \mathrm{I}_{\mathrm{dia}}$ 
a

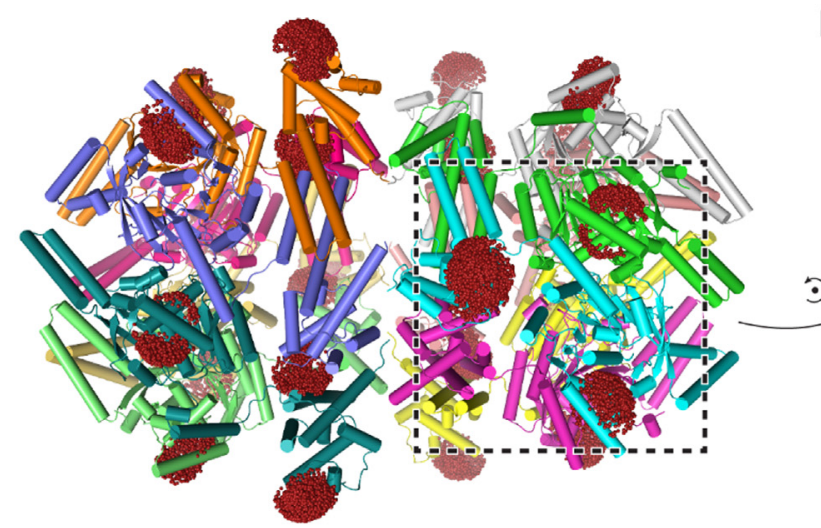

c

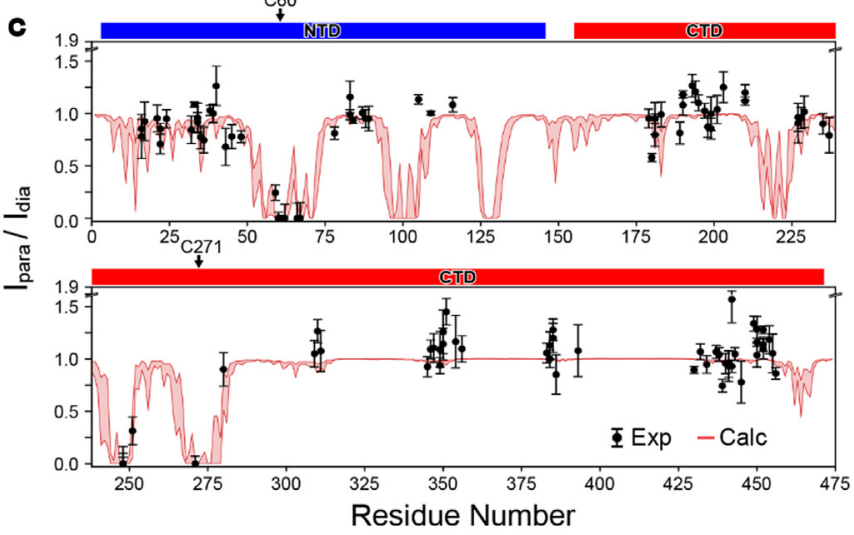

b

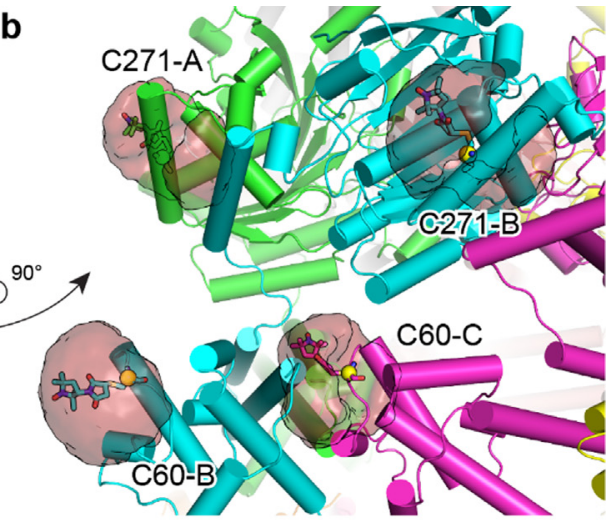

d

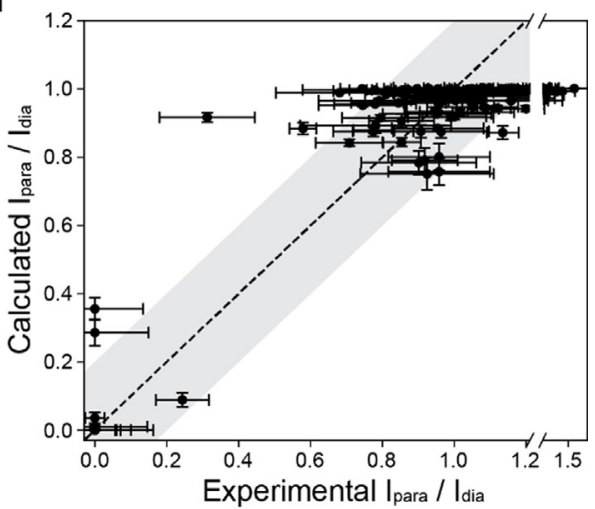

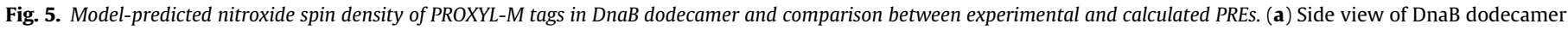

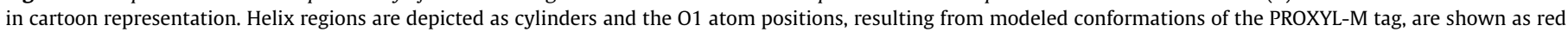

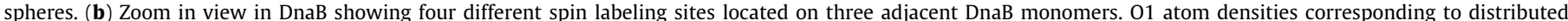

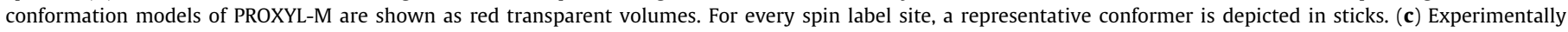

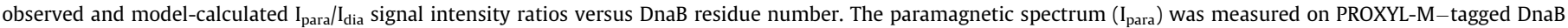

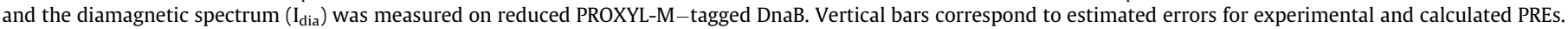

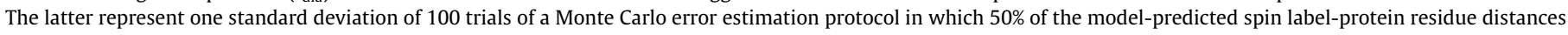

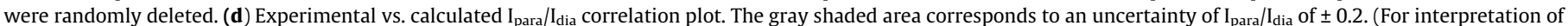
the references to color in this figure legend, the reader is referred to the web version of this article.)

values with deviations from the calculated curve clustered around residue positions 400 and 450 in Fig. 6c. This could possibly indicate flexibility of the CTD which also escaped detection by solidstate NMR. Another small but noticeable difference between experimentally observed and calculated $\mathrm{I}_{\text {para }} / \mathrm{I}_{\mathrm{dia}}$ values was found for the cross peaks of V34. This could indicate increased conformational flexibility of the helix $\alpha 1-\alpha 2$-connecting loop containing V34. Alternatively, this could indicate some flaws of the X-ray structure model in this loop region, which is poorly defined by the previously reported low-resolution electron density (see for a further discussion the supporting material and Figure S6).

Interestingly, this discrepancy between measured and theoretical PREs for V34 is not observed for the PROXYL-M-labeled DnaB sample (Fig. 5d), which could indicate differences in the flexibility of the two tags. Alternatively, these distinct behaviors could be caused by the different sizes of the blind spheres of $\mathrm{Gd}^{3+}$-DOTA$\mathrm{M}$ and PROXYL-M tags (see below). For PROXYL-M-tagged DnaB, the side-chain of V34 is $\sim 15 \AA$ away from the 01 atom of PROXYL-M, thus clearly outside the blind sphere for this tag (Fig. 7a). By contrast, for $\mathrm{Gd}^{3+}$-DOTA-M-tagged DnaB, V34 is near the surface of the blind sphere at a distance ( $\sim 16 \AA$ from $\mathrm{Gd}^{3+}$ ion) that corresponds to a region in the intensity-vs-spin label distance curve with the steepest slope (Fig. 7b). Thus, small distance changes for V34, caused by flexibility of the tag or mobility of the loop comprising residue $\mathrm{V} 34$, could result in more pronounced PRE changes for $\mathrm{Gd}^{3+}$-DOTA-M than for PROXYL-M.

\subsection{Determination of the blind spheres of $G d^{3+}$-DOTA-M and PROXYL- M spin labels}

An important consideration for NMR experiments employing paramagnetic spin labels is the size of the so-called "blind sphere", i.e., the region where a nucleus is sufficiently close to the unpaired electron so that its NMR signal is broadened entirely beyond detection. To determine the blind spheres induced by the nitroxide radical and $\mathrm{Gd}^{3+}$ ion for direct detection of ${ }^{13} \mathrm{C}$, we plotted the experimental PRE values versus the model-determined effective nitroxide-carbon and $\mathrm{Gd}^{3+}$-carbon distances, respectively, and analyzed the resultant curves by calculating the least-squares fits to the theoretically-derived equation described in reference [18]. Fig. 7 and Figures S7 and S8 illustrate how the PRE $\left(\mathrm{I}_{\text {para }} / \mathrm{I}_{\text {dia }}\right)$ changes with the nitroxide-carbon or $\mathrm{Gd}^{3+}$-carbon distance in the 2D DARR and 3D NCACB experiment, respectively.

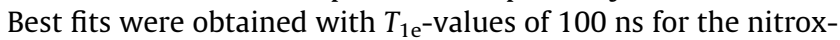
ide radical and $20 \mathrm{~ns}$ for the $\mathrm{Gd}^{3+}$ ion, which agrees well with previously determined values for $T_{1 \mathrm{e}}$ [62]. Assuming a lower detection limit for $I_{\text {para }} / I_{\text {dia }}$ of $25 \%$, below which an NMR signal is usually too weak to be detected in the paramagnetic spectrum, these fits yielded estimates for the size of the ${ }^{13} \mathrm{C}$ blind sphere in 2D DARR spectra of $11 \AA$ and $14 \AA$ for the nitroxide and $\mathrm{Gd}^{3+}$, respectively. In comparison, the nitroxide tag leads to a slightly larger ${ }^{13} \mathrm{C}$ blind sphere in the 3D NCACB spectrum of $12 \AA$ (Figure S5c and S8). These values compare favorably with the reported ones for the 
a

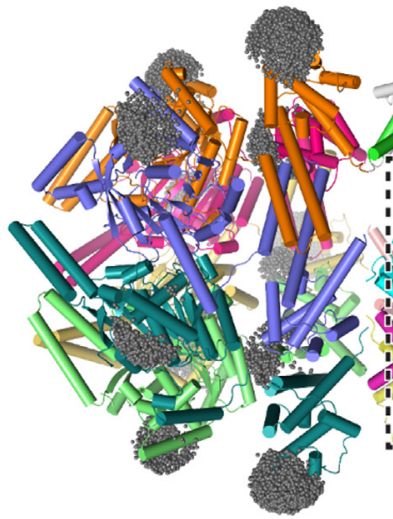

c

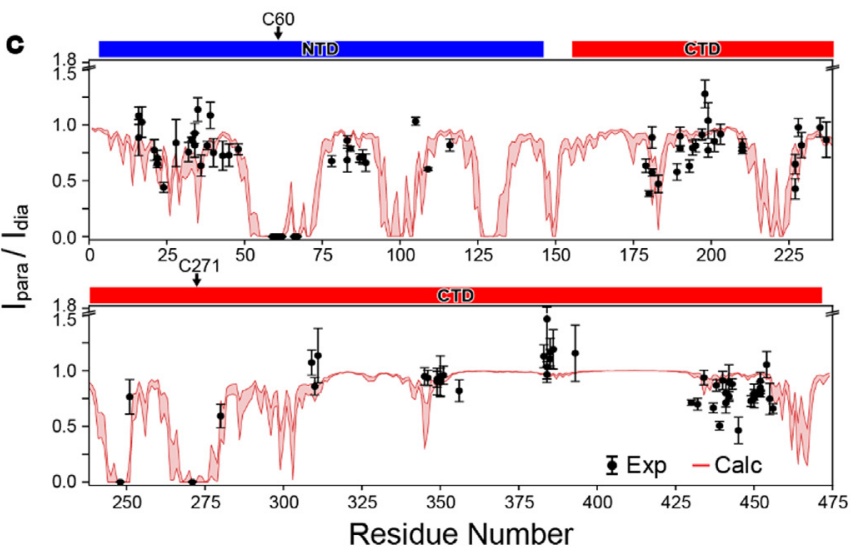

b

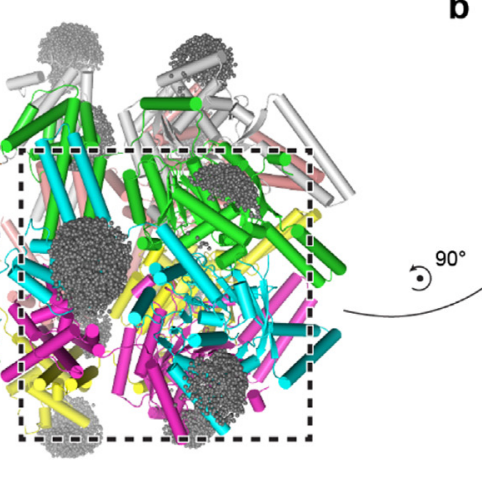

d

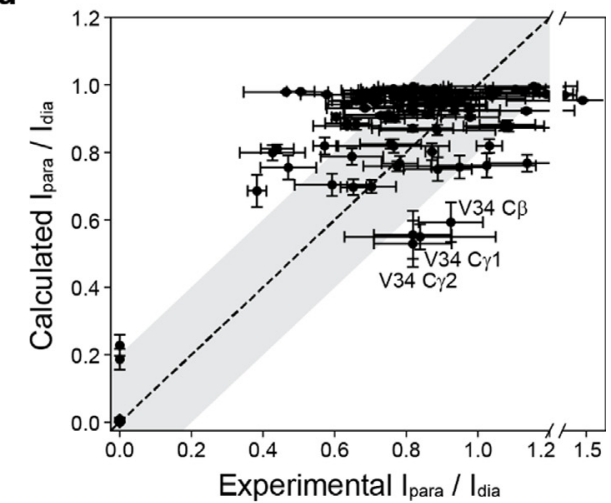

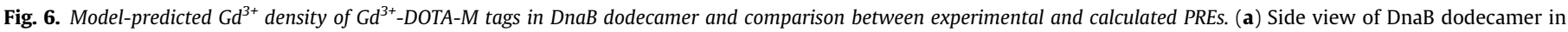

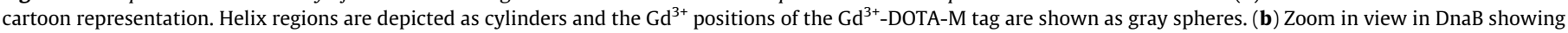

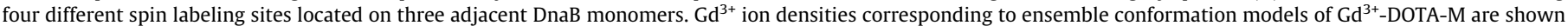

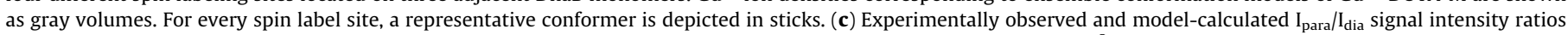

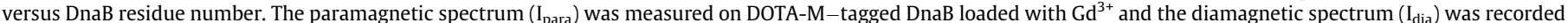

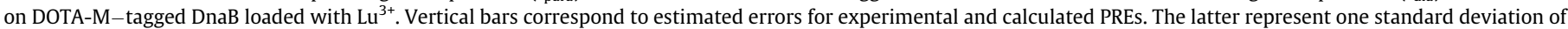

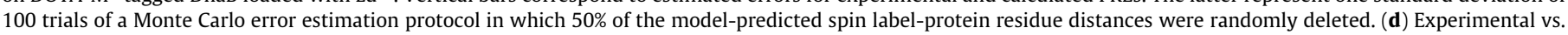
calculated $\mathrm{I}_{\mathrm{para}} / \mathrm{I}_{\mathrm{dia}}$ correlation plot. The gray shaded area corresponds to an uncertainty of $\mathrm{I}_{\text {para }} / \mathrm{I}_{\mathrm{dia}}$ of \pm 0.2 .

a

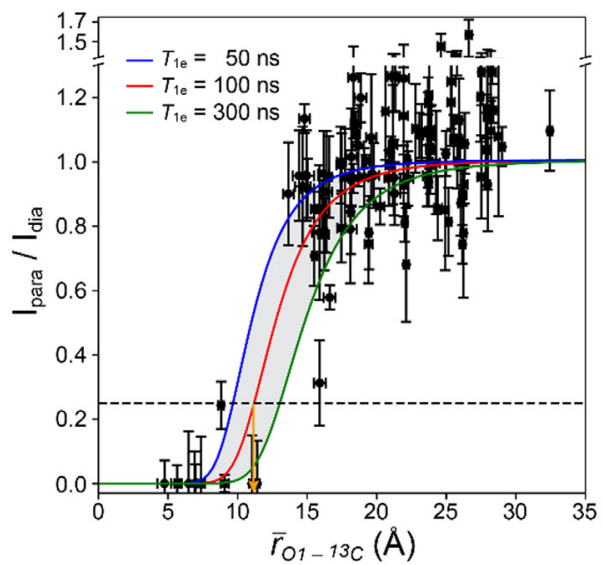

b

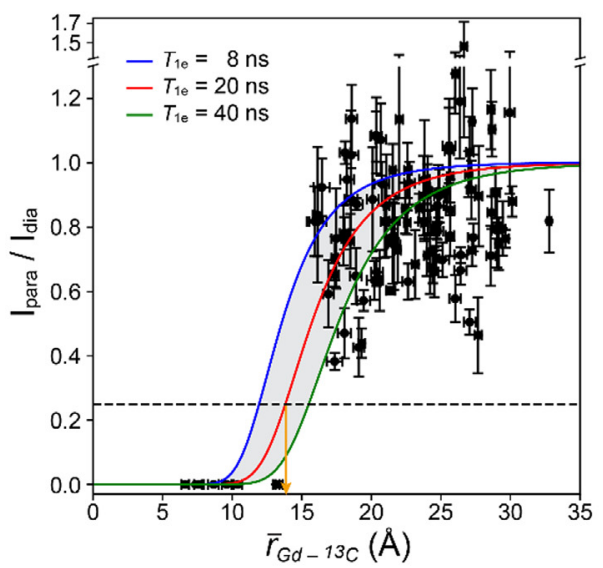

C

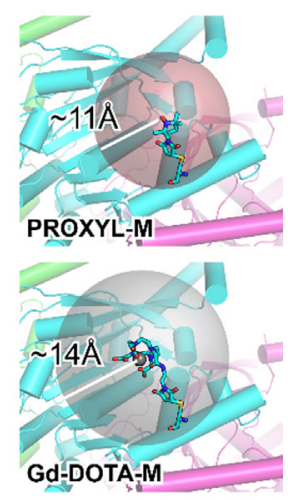

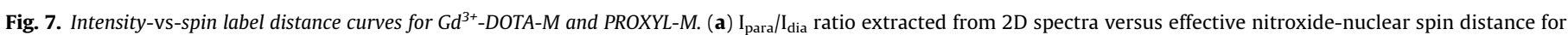

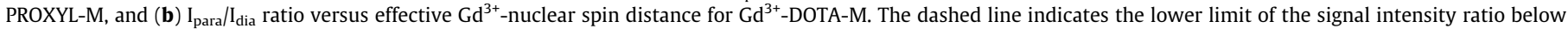

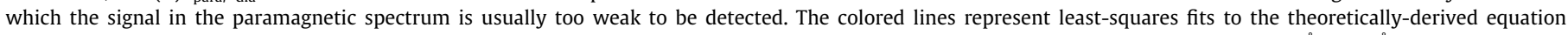

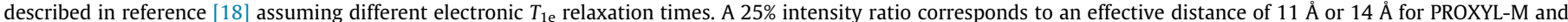

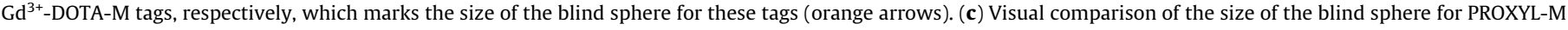
and Gd-DOTA-M tags in DnaB. (For interpretation of the references to color in this figure legend, the reader is referred to the web version of this article.) 
a

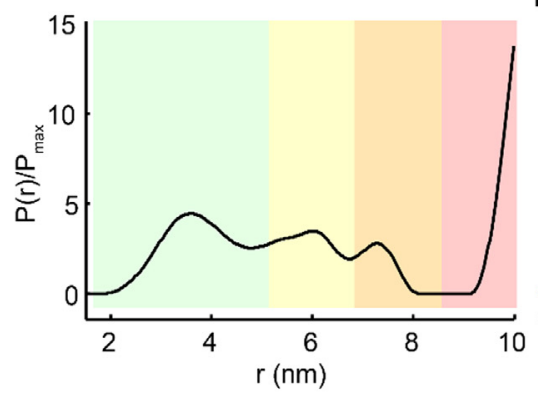

b

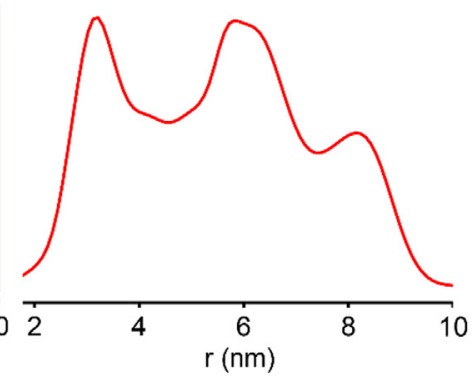

c

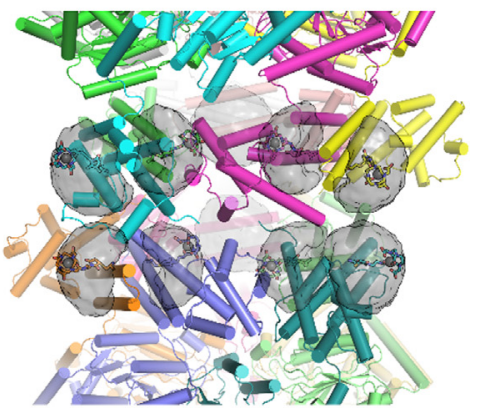

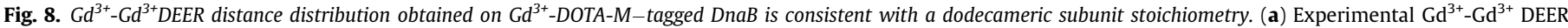

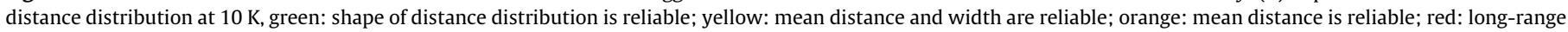

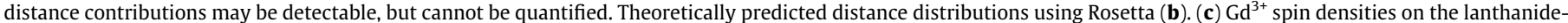

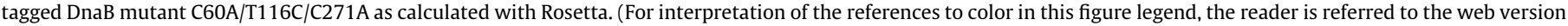
of this article.)

nitroxide in MTSL [63] or the $\mathrm{Gd}^{3+}$ ion in dopants and metalloproteins [64,65], as well as in metal-ion DNP studies employing $\mathrm{Gd}^{3+}$-DOTA-M tags [26]. Noteworthy, the lengths of the linker connecting the protein backbone to the nitroxide $(10.2 \AA)$ or the $\mathrm{Gd}^{3+}$ ion $(12.5 \AA)$ in the model structures is only slightly smaller than the size of the blind sphere determined in Fig. 7. A sufficiently large linker ensures that the unpaired electron is removed from the protein surface, and, thus, avoids that too many residues get within the blind region of the spin label, which is relevant for instance in protein-protein interaction studies. If, however, the blind sphere information shall be used in structure determination, tags with shorter linkers are even favored.

\subsection{EPR distance measurements using $\mathrm{Gd}^{3+}$-DOTA-M-tagged DnaB}

Spin labeled DnaB also opens the way for EPR distance measurements using dipolar electron-electron resonance (DEER) spectroscopy [66-68]. We recorded $\mathrm{Gd}^{3+}-\mathrm{Gd}^{3+}$ DEER [69] data after labeling DnaB with $\mathrm{Gd}^{3+}$-DOTA-M at a single cysteine site. To this end, residue T116 was replaced by a cysteine and the two native cysteine residues, C60 and C271, were mutated to an alanine and a serine, respectively. The DEER distance distribution in Fig. 8a (for the primary data see Figure S9) shows three maxima at around $3.8,6$, and $7.5 \mathrm{~nm}$, although the latter cannot be extracted with high confidence. We compared the location of the maxima in the experimental distance distribution to the ones in the distance distribution obtained by molecular modeling with Rosetta (Fig. 8b) and found a good overall agreement. Such a distance distribution is consistent with a labeling of all available T116C sites in the dodecameric DnaB crystal structure model (Fig. 8c) and therefore serves as spectroscopic evidence that DnaB was present as a dimer of hexamers (or dodecameric assembly). This is further supported by the less good agreement between the experimental distance distribution and the simulated one based on only a hexameric assembly (see Figure S10). Similar experiments will also allow us in the future to probe interactions of DnaB with other proteins, such as with the DnaG primase within the primosome complex.

\section{Conclusions}

We herein describe the site-directed covalent spin labeling of the bacterial DnaB helicase from Helicobacter pylori with PROXYL$\mathrm{M}$ and DOTA-M tags. DnaB constitutes a large protein with 488 residues per monomer and we show that paramagnetic solidstate NMR on this oligomeric assembly becomes possible opening the way for structural investigations. Site-specific PRE data were extracted from 2D and 3D solid-state NMR spectra and agreed well with PREs back-calculated from the DnaB structure by computational modeling using the low-resolution DnaB crystal structure. The radius of the blind sphere of PROXYL-M in ${ }^{13} \mathrm{C}-{ }^{13} \mathrm{C}$ DARR experiments $(\sim 11 \AA)$ is smaller than that of $\mathrm{Gd}^{3+}$-DOTA-M ( $\sim 14 \AA)$. This information will be of practical relevance for the design of future NMR studies using these paramagnetic tags. While the quantitative interpretation of PREs became only possible due to the availability of a crystal structure for DnaB, we envision that in the future paramagnetic labeling of DnaB using the described approaches will provide new structural insights into DnaB and its interactions, for instance in the primosome complex, which is formed between DnaB and its DnaG primase. Our study showcases the combined application of paramagnetic spin labeling, solid-state NMR, and computational modeling for the structural characterization of the large DnaB complex. Integrative technologies like these have the potential to facilitate the structure determination of biomolecular systems that are difficult to study by only a single technique, such as flexible and dynamic macromolecular assemblies and membrane-associated complexes. We also imagine that EPR experiments on spin labeled DnaB will be a useful tool providing new insights into the mode of action of DnaB and for large motor proteins in general.

\section{Materials and methods}

\subsection{Sample preparation}

${ }^{13} \mathrm{C}$ and ${ }^{15} \mathrm{~N}$ labeled DnaB was expressed as described in reference [70]. All protein solutions were sedimented [58,71,72] in the MAS-NMR rotor $\left(16 \mathrm{~h}\right.$ at $4{ }^{\circ} \mathrm{C}$ at $\left.210,000 \times \mathrm{g}\right)$ using homebuilt tools [73].

\subsubsection{Tagging of DnaB with PROXYL-M}

DnaB solution (20 mg dissolved in $5 \mathrm{~mL}$ buffer $(2.5 \mathrm{mM}$ sodium phosphate, $\mathrm{pH} 7.5,130 \mathrm{mM} \mathrm{NaCl}$ )) was transferred to new buffer (10 mM sodium phosphate, $150 \mathrm{mM} \mathrm{NaCl}, 10 \%$ glycerine, $\mathrm{pH} 7.5$ ) using PD-10 column. The protein was concentrated to $40 \mathrm{mg} / \mathrm{mL}$ by centrifugation using Millipore concentrator (30 kDa cut-off). The spin labeling reaction with PROXYL-M was performed at $4{ }^{\circ} \mathrm{C}$ and $\mathrm{pH} 7.5$ for $18 \mathrm{~h}$ using a protein concentration of $50 \mu \mathrm{M}$ and a 30 -fold excess of spin label (per cysteine). The excess of spin label was removed with PD-10 column.

\subsubsection{Tagging of DnaB with the reduced form of PROXYL-M}

DnaB solution (32 mg dissolved in $10 \mathrm{~mL}$ buffer $(2.5 \mathrm{mM}$ sodium phosphate, $\mathrm{pH} 7.5,130 \mathrm{mM} \mathrm{NaCl})$ ) was transferred to new buffer (10 mM sodium phosphate, $150 \mathrm{mM} \mathrm{NaCl}, 10 \%$ 
glycerine, $\mathrm{pH}$ 7.5) using $\mathrm{PD}-10$ column. The protein was concentrated to $7 \mathrm{mg} / \mathrm{mL}$ by centrifugation using Millipore concentrator (30 kDa cut-off). The spin labeling reaction with PROXYL-M was performed at $4{ }^{\circ} \mathrm{C}$ and $\mathrm{pH} 7.5$ for $18 \mathrm{~h}$ using a protein concentration of $110 \mu \mathrm{M}$ and a 30 -fold excess of spin label (per cysteine). The reduction of the PROXYL-M tag was performed in a new buffer (10 mM sodium phosphate, $150 \mathrm{mM} \mathrm{NaCl}, 30 \mathrm{mM}$ ascorbic acid, $10 \%$ glycerine, $\mathrm{pH} 7.5)[22]$. The excess of spin label was removed with PD-10 column.

\subsubsection{Tagging of DnaB with $\mathrm{Lu}^{3+}$-DOTA-M}

DnaB solution ( $35 \mathrm{mg}$ dissolved in $9 \mathrm{~mL}$ buffer $(2.5 \mathrm{mM}$ sodium phosphate, $\mathrm{pH} 7.5,130 \mathrm{mM} \mathrm{NaCl})$ ) was transferred to new buffer (10 mM sodium phosphate, $150 \mathrm{mM} \mathrm{NaCl}, 10 \%$ glycerine, $\mathrm{pH} 7.5$ ) using PD-10 column. The protein was concentrated to $15 \mathrm{mg} / \mathrm{mL}$ by centrifugation using Millipore concentrator (30 kDa cut-off). The spin labeling reaction with $\mathrm{Lu}^{3+}$-DOTA-M was performed at $4{ }^{\circ} \mathrm{C}$ and $\mathrm{pH} 7.5$ for $18 \mathrm{~h}$ using a protein concentration of $160 \mu \mathrm{M}$ and a 30 -fold excess of spin label (per cysteine). The excess of spin label was removed with PD-10 column.

\subsubsection{Tagging of DnaB with $\mathrm{Gd}^{3+}$-DOTA-M}

DnaB solution (35 mg dissolved in $9 \mathrm{~mL}$ buffer $(2.5 \mathrm{mM}$ sodium phosphate, $\mathrm{pH} 7.5,130 \mathrm{mM} \mathrm{NaCl})$ ) was transferred to new buffer (10 mM sodium phosphate, $150 \mathrm{mM} \mathrm{NaCl}, 10 \%$ glycerine, $\mathrm{pH} 7.5$ ) using PD-10 column. The protein was concentrated to $15 \mathrm{mg} / \mathrm{mL}$ by centrifugation using Millipore concentrator (30 kDa cut-off). The spin labeling reaction with $\mathrm{Gd}^{3+}$-DOTA-M was performed at $4{ }^{\circ} \mathrm{C}$ and $\mathrm{pH} 7.5$ for $18 \mathrm{~h}$ using a protein concentration of $160 \mu \mathrm{M}$ and a 30 -fold excess of spin label (per cysteine). The excess of spin label was removed with PD-10 column.

\subsubsection{EPR sample preparation}

DnaB with PROXYL-M. DnaB with PROXYL-M solution (1.09 mg dissolved in $25 \mu \mathrm{L}$ buffer ( $10 \mathrm{mM}$ sodium phosphate, $150 \mathrm{mM} \mathrm{NaCl}$, $10 \%$ glycerine, $\mathrm{pH} 7.5)$ ) was used for the CW-EPR experiment.

PROXLY-M reference solution. A 3.4 mM PROXYL-M stock solution in buffer (10 mM sodium phosphate, $150 \mathrm{mM} \mathrm{NaCl}, 10 \%$ glycerine, $\mathrm{pH}$ 7.5) was prepared. The solution was diluted with buffer (10 mM sodium phosphate, $150 \mathrm{mM} \mathrm{NaCl}, 10 \%$ glycerine, $\mathrm{pH} 7.5$ ) until a concentration of $100 \mu \mathrm{M}$ PROXYL-M was reached.

DnaB-C60A C271S T116C with Gd ${ }^{3+}$-DOTA-M. DnaB with $\mathrm{Gd}^{3+}-$ DOTA-M solution $(0.25 \mathrm{mg}$ dissolved in $0.5 \mathrm{~mL}$ buffer $(10 \mathrm{mM}$ sodium phosphate, $\mathrm{pH} 7.5,150 \mathrm{mM} \mathrm{NaCl}$ )) was transferred to new buffer (10 mM sodium phosphate, $150 \mathrm{mM} \mathrm{NaCl}, 10 \%$ glycerine, $\mathrm{pH} 7.5$ ) and concentrated to $2.5 \mathrm{mg} / \mathrm{mL}$ by centrifugation using Millipore concentrator (10 kDa cut-off). DnaB solution was transferred to new buffer (10 mM sodium phosphate, $150 \mathrm{mM} \mathrm{NaCl}$, $\mathrm{pH} 7.8, \mathrm{D}_{2} \mathrm{O}$ ) and concentrated to $3.6 \mathrm{mg} / \mathrm{mL}$ by centrifugation using Millipore concentrator (10 kDa cut-off). The mutant C60A C271S T116 was expressed as described above for the wild-type protein.

\subsection{Solid-state NMR}

Solid-state NMR spectra were acquired on a wide-bore $850 \mathrm{MHz}$ Bruker Avance III spectrometer using a $3.2 \mathrm{~mm}$ Bruker Biospin "Efree" probe [74]. The MAS frequency was set to $17.0 \mathrm{kHz}$ and the sample temperature was adjusted to $278 \mathrm{~K}$ using the water line as an internal reference [73]. The 2D spectra were processed with the software TOPSPIN (version 3.5, Bruker Biospin) with a shifted (3.0) squared cosine apodization function and automated baseline correction in the indirect and direct dimension. Spectral analysis was performed using CcpNmr Analysis 2.4.2 [75-77]. The spectra were referenced to 4,4-dimethyl-4-silapentane-1-sulfonic acid (DSS). For all experimental details see Table S4.

\section{3. $E P R$}

\subsubsection{Continuous-wave EPR spectroscopy}

CW EPR spectra of nitroxide spin labeled protein were recorded at room temperature at $\mathrm{X}$ band $(9.5 \mathrm{GHz})$ using a Bruker ElexsysE500 spectrometer including a Bruker super high $Q$ resonator ER4122SHQ. Protein samples were filled into $50 \mu \mathrm{L}$ glass capillaries with an outer/inner diameter of $1.5 / 0.9 \mathrm{~mm}$ (BLAUBRANDs). All spectra were recorded with $100 \mathrm{kHz}$ field modulation, $1 \mathrm{G}$ modulation amplitude, a time constant of $10.24 \mathrm{~ms}$, a conversion time of $40.96 \mathrm{~ms}$ and $25 \mathrm{~dB}$ microwave power attenuation. Spin labeling efficiencies were calculated by digital double integration of the recorded spectra and comparison with the reference EPR measurement of nitroxide solution of $100 \mu \mathrm{M}$ concentration.

\subsubsection{DEER spectroscopy}

Pulsed EPR measurements were performed at a home-built high power Q-band spectrometer ( $35 \mathrm{GHz}$ ) with $200 \mathrm{~W}$ output microwave power, a Bruker ElexSys acquisition system (E580) and a home-built TE001 pulse probe (OD $3 \mathrm{~mm}$ sample tubes). Temperature stabilisation during all measurements was performed with a He-flow cryostat (ER $4118 \mathrm{CF}$, Oxford Instruments) and a temperature control system (ITC 503, Oxford Instruments). The four pulse DEER experiment was used to measure $\mathrm{Gd}^{3+}-\mathrm{Gd}^{3+}$ distances at $10 \mathrm{~K}$. The frequency offset between detection and pump pulse was $100 \mathrm{MHz}$, all pulse lengths were set to $12 \mathrm{~ns}$, the primary echo delay time was set to $400 \mathrm{~ns}$, with pump pulse step of $12 \mathrm{~ns}$ and an eight-step ESEEM averaging cycle with a step of 8 ns. Intermolecular couplings, which cause background decay in the time domain data, were removed by background correction (with dimension parameter $\mathrm{D}=3.0$ ) and distance distributions in a range of 1.5 to $10.0 \mathrm{~nm}$ were obtained by Tikhonov regularization.

\subsection{Structural modeling of Gd-DOTA-M and PROXYL-M tags in DnaB}

The structures of Gd-DOTA-M and PROXYL-M tags conjugated to the side-chain of cysteine (termed Gd-DOTA-M-Cys or PROXYL-M-Cys, respectively) were built in Avogadro (version1.2)[78]. Gd-DOTA-M-Cys was built in two absolute configurations ( $R$ and $S$ ) to account for the presence of a chiral carbon atom in the Cys-linked maleimide ring. For PROXYL-M-Cys, the structures of four stereoisomers were considered in molecular modeling because of two chiral atoms, one at the maleimide ring and another at the PROXYL ring (see Figure S4). Structure geometry was optimized by DFT calculations with Gaussian09 (Gaussian, Inc., Wallingford $\mathrm{CT}$ ) using the quasi-relativistic effective core potential (ECP)[79] and [5s4p3d]-GTO valence basis sets for the $\mathrm{Gd}^{3+}$ ion, the 6-31(d,p) standard basis set for all other atoms, and the M06 functional [80]. Solvation effects were evaluated with the integral equation formalism of the polarizable continuum model (IEFPCM)[81] implemented in Gaussian09.

The BCL::ConformerGenerator method [82] was used to build libraries of 2000 unique conformations for each stereoisomer of Gd-DOTA-M-Cys and PROXYL-M-Cys, respectively. The method generates $3 \mathrm{D}$ ligand conformers by combining rotamers of small molecule structures from the Crystallography Open Database (COD) $[60,61]$ according to a statistically-derived scoring function. For Gd-DOTA-M-Cys, the linker connecting the DOTA chelator to the backbone $\mathrm{C} \alpha$ atom was fully flexible in conformer generation, while the Gd-DOTA complex was treated as rigid. For PROXYL$\mathrm{M}$-Cys, all four side-chain dihedral angles were flexible. A total of 20,000 conformer generation iterations were carried out from which the 2000 best-scoring conformations were kept after removing similar conformers with a pairwise root-mean-squared distance deviation (RMSD) $<0.25 \AA$. The resulting conformer libraries were deemed nearly complete as all of the expected 
rotamers of the six (Gd-DOTA-M-Cys) or four (PROXYL-M-Cys) dihedral angles occurred with similar probabilities (see Figure S4).

The Rosetta software (version 3.12)[55] was used to model the side-chain conformations and determine the spin density for GdDOTA-M and PROXYL-M at every cysteine residue in DnaB. First, the structure of dodecameric DnaB from H. pylori (PDB: 4ZC0) [54] was refined by adding missing residues, which were not resolved in the original X-ray structure model, and performing constrained minimization in the Rosetta force field [83]. Modeling of unresolved regions was accomplished by insertion of backbone fragments with PSIPRED-[84] and JUFO9D-[85] predicted secondary structures in combination with cyclic coordinate descent (CCD)[86] loop building algorithms implemented through the Rosetta comparative modeling (RosettaCM)[87] program. In this step, D3 symmetry of DnaB dodecamer was enforced with the help of symmetry definition files [88], and $\mathrm{C} \alpha$-atom pair distance constraints were applied to prevent any large movements of the protein backbone during loop modeling and minimization. Second, all of the 24 native cysteine residues in DnaB were successively replaced by every conformer of the $\mathrm{S}$ - and R-isomers of $\mathrm{Gd}$ DOTA-M-Cys or PROXYL-M-Cys, respectively, by aligning the corresponding backbone atoms of protein and spin label residue with each other. The backbone and side-chain degrees of freedom of all protein residues surrounding the spin label were minimized using the Rosetta all-atom ref2015 energy function [83] while applying weak distance constraints between all pairs of $\mathrm{C} \alpha$-atoms which were within $10 \AA$ of each other. Spin label conformers which still clashed with the protein after minimization according to a Rosetta energy cutoff $(-5000 \mathrm{kcal} / \mathrm{mol})$ were removed. The remaining conformers were used to calculate the spin density distribution for the $\mathrm{Gd}^{3+}$ ion or the nitroxide radical, respectively. The molecular surface area of cysteine residues in the DnaB structure model was measured with the get_area function in PyMOL (The PyMOL Molecular Graphis System, Version 2.4, Schrödinger, LCC).

\subsection{Simulation of PREs for $\mathrm{Gd}^{3+}$ and nitroxide spin labels}

The intensity ratio of an NMR signal recorded in the para- vs. diamagnetic spectrum was calculated according to:

$\frac{I_{\text {para }}}{I_{\text {dia }}}=I_{C P}\left(\tau_{C P}\right) \cdot I_{t 1} \cdot I_{D A R R}\left(\tau_{D A R R}\right) \cdot I_{t 2}$

where $I_{C P}$ is the change in signal intensity during the cross polarization (CP) step, $\mathrm{I}_{\mathrm{DARR}}$ is the signal reduction during the DARR mixing period, and $\mathrm{I}_{\mathrm{t} 1}$ and $\mathrm{I}_{\mathrm{t} 2}$ represent the signal intensity reduction during the evolution $\left(t_{1}\right)$ and detection $\left(t_{2}\right)$ periods, respectively. The derivation of Eq. (3) is described in detail in Tamaki et al. [18]. $\mathrm{I}_{\mathrm{CP}}$ is dependent on the paramagnetic relaxation rates of ${ }^{1} \mathrm{H}$ and ${ }^{13} \mathrm{C}$ in the rotating frame $\left(\Gamma_{1 \rho, \mathrm{H}}, \Gamma_{1 \rho, \mathrm{C}}\right)$, I $\mathrm{DARR}_{\mathrm{DAR}}$ is a function of the paramagnetic longitudinal relaxation rate of ${ }^{13} \mathrm{C}\left(\Gamma_{1, \mathrm{C}}\right)$, and $\mathrm{I}_{\mathrm{t} 1}$ and $\mathrm{I}_{\mathrm{t} 2}$ are determined by the paramagnetic transversal relaxation rate of ${ }^{13} \mathrm{C}\left(\Gamma_{2, \mathrm{C}}\right)$. All equations and simulation parameters are provided in the supporting material (see Figures S7, S8 and S11).

The effective unpaired electron-nucleus distance, $\bar{r}$, was calculated based on the refined DnaB structure model and the simulated spin density distributions for $\mathrm{Gd}^{3+}$ and the nitroxide radical according to [26]:

$\bar{r}=\left(\frac{1}{N_{\text {chains }}} \sum_{i=1}^{N_{\text {chains }}} \sum_{j=1}^{N_{S L}} r_{i j}^{-6}\right)^{-\frac{1}{6}}$

$N_{\mathrm{SL}}$ is the number of spin label sites $\left(N_{\mathrm{SL}}=24\right)$ in the DnaB dodecamer, and $r_{\mathrm{ij}}$ is the distance between a carbon atom in DnaB, for which a cross peak was detected in the NMR experiment, and the center of mass of the $\mathrm{Gd}^{3+}$ ion (DOTA-M) or 01 atom (PROXYL-M) positions, respectively, for each of the 24 spin label sites. In calculating the center of mass of the spin density, we assumed an $\mathrm{N}$-jump model for the spin label in accordance with previous approaches $[59,89]$. Because the dipolar relaxation of a nucleus in one DnaB monomer is the combined effect of all 24 spin labels in DnaB dodecamer, the effective distance in equation (4) is evaluated by the sum of $\mathrm{r}^{-6}$ distances to the $\mathrm{Gd}^{3+}$ or $\mathrm{O} 1$ centers of mass of all 24 spin label sites. In addition, the distances to both DnaB carbon atoms, which contribute to the same NMR cross peak, are included in the summation in Eq. (4). Note that because the conformations of chains A and B in the DnaB crystal structure are not identical ( $C \alpha$-RMSD is $4.6 \AA$ over $72 \%$ of the residues), $\mathrm{r}^{-6}$ averaging of the distances calculated for chains $A$ and $B$ is additionally applied in Eq. (4). Thus, $N_{\text {chains }}$ denotes the number of asymmetric protein chains (A and B, $N_{\text {chains }}=2$ ) in the DnaB dodecamer.

\section{Declaration of Competing Interest}

The authors declare that they have no known competing financial interests or personal relationships that could have appeared to influence the work reported in this paper.

\section{Acknowledgements}

This work was supported by the ETH Research Grant ETH-43 17-2 (T.W.) and the Deutsche Forschungsgemeinschaft (DFG, German Research Foundation, project number 455240421 and Heisenberg fellowship, project number 455238107, T.W.). GK was supported by a DFG postdoctoral fellowship (KU 3510/1-1). TW acknowledges helpful discussions with Prof. Beat H. Meier (ETH Zürich, Switzerland), Dr. Anja Böckmann and Dr. Laurent Terradot (both MMSB Lyon, France) as well as Dr. Carole Gardiennet (Université de Lorraine, France). Prof. Beat H. Meier is also acknowledged for providing NMR measurement time.

\section{Appendix A. Supplementary material}

Supplementary data to this article can be found online at https://doi.org/10.1016/j.jmr.2021.107075. Rosetta input files to simulate conformer ensembles of DOTA and PROXYL tags as well as $R$ scripts to simulate signal decay curves for 2D DARR and 3D NCACB experiments are available under 10.5281/zenodo.5515550.

\section{References}

[1] M. Bonaccorsi, T. Le Marchand, G. Pintacuda, Protein structural dynamics by Magic-Angle Spinning NMR, Curr. Opin. Struct. Biol. 70 (2021) 34-43.

[2] P.C.A. van der Wel, New applications of solid-state NMR in structural biology, Emerg. Top Life Sci. 2 (2018) 57-67.

[3] E. Nimerovsky, K.T. Movellan, X.C. Zhang, M.C. Forster, E. Najbauer, K. Xue, R. Dervişoğlu, K. Giller, C. Griesinger, S. Becker, L.B. Andreas, Proton Detected Solid-State NMR of Membrane Proteins at 28 Tesla $(1.2 \mathrm{GHz})$ and $100 \mathrm{kHz}$ Magic-Angle Spinning, Biomolecules 11 (2021) 752.

[4] M. Callon, A.A. Malär, S. Pfister, V. Římal, M.E. Weber, T. Wiegand, J. Zehnder, M. Chávez, R. Cadalbert, R. Deb, A. Däpp, M.-L. Fogeron, A. Hunkeler, L. Lecoq, A Torosyan, D. Zyla, R. Glockshuber, S. Jonas, M. Nassal, M. Ernst, A. Böckmann, B. H. Meier, Biomolecular solid-state NMR spectroscopy at $1200 \mathrm{MHz}$ : the gain in resolution, J. Biomol. NMR, (2021).

[5] A. Böckmann, M. Ernst, B.H. Meier, Spinning proteins, the faster, the better?, J Magn. Reson. 253 (2015) 71-79.

[6] A. Samoson, H-MAS, J. Magn. Reson. 306 (2019) 167-172.

[7] B. Habenstein, C. Wasmer, L. Bousset, Y. Sourigues, A. Schuetz, A. Loquet, B.H. Meier, R. Melki, A. Böckmann, Extensive de novo solid-state NMR assignments of the $33 \mathrm{kDa}$ C-terminal domain of the Ure2 prion, J. Biomol. NMR 51 (2011) 235-243.

[8] Y. Shen, F. Delaglio, G. Cornilescu, A. Bax, TALOS+: a hybrid method for predicting protein backbone torsion angles from NMR chemical shifts, J. Biomol. NMR 44 (2009) 213-223.

[9] G. Pintacuda, G. Kervern, Paramagnetic Solid-State Magic-Angle Spinning NMR Spectroscopy, in: H. Heise, S. Matthews (Eds.), Modern NMR Methodology, Springer, Berlin Heidelberg, 2013, pp. 157-200. 
[10] G. Otting, Protein NMR Using Paramagnetic Ions, Ann. Rev. Biophys. 39 (2010) 387-405.

[11] G. Otting, Prospects for lanthanides in structural biology by NMR, J. Biomol. NMR 42 (2008) 1-9.

[12] C.P. Jaroniec, Solid-state nuclear magnetic resonance structural studies of proteins using paramagnetic probes, Solid State Nucl. Magn. Reson. 43-44 (2012) 1-13.

[13] G.M. Clore, J. Iwahara, Theory, Practice, and Applications of Paramagnetic Relaxation Enhancement for the Characterization of Transient Low-Population States of Biological Macromolecules and Their Complexes, Chem. Rev. 109 (2009) 4108-4139.

[14] A.J. Pell, G. Pintacuda, C.P. Grey, Paramagnetic NMR in solution and the solid state, Prog. Nucl. Magn. Reson. Spectrosc. 111 (2019) 1-271.

[15] I. Solomon, Relaxation Processes in a System of Two Spins, Phys. Rev. 99 (1955) $559-565$.

[16] N. Bloembergen, L.O. Morgan, Proton Relaxation Times in Paramagnetic Solutions. Effects of Electron Spin Relaxation, J. Chem. Phys. 34 (1961) 842-850.

[17] C.P. Jaroniec, Structural studies of proteins by paramagnetic solid-state NMR spectroscopy, J. Magn. Reson. 253 (2015) 50-59.

[18] H. Tamaki, A. Egawa, K. Kido, T. Kameda, M. Kamiya, T. Kikukawa, T. Aizawa, T. Fujiwara, M. Demura, Structure determination of uniformly 13C, 15N labeled protein using qualitative distance restraints from MAS solid-state 13C-NMR observed paramagnetic relaxation enhancement, J. Biomol. NMR 64 (2016) 87-101.

[19] S. Balayssac, I. Bertini, M. Lelli, C. Luchinat, M. Maletta, Paramagnetic Ions Provide Structural Restraints in Solid-State NMR of Proteins, J. Am. Chem. Soc. 129 (2007) 2218-2219.

[20] J. Zehnder, R. Cadalbert, L. Terradot, M. Ernst, A. Bockmann, P. Guntert, B.H. Meier, T. Wiegand, Paramagnetic Solid-State NMR to Localize the Metal-Ion Cofactor in an Oligomeric DnaB Helicase, Chemistry 27 (2021) 7745-7755.

[21] T. Wiegand, D. Lacabanne, K. Keller, R. Cadalbert, L. Lecoq, M. Yulikov, L. Terradot, G. Jeschke, B.H. Meier, A. Böckmann, Solid-state NMR and EPR Spectroscopy of Mn2+-Substituted ATP-Fueled Protein Engines, Angew. Chem. Int. Ed. 56 (2017) 3369-3373.

[22] M. Ahmed, A. Marchanka, T. Carlomagno, Structure of a Protein-RNA Complex by Solid-State NMR Spectroscopy, Angew. Chem. Int. Ed. Engl. 59 (2020) 68666873.

[23] P.S. Nadaud, J.J. Helmus, S.L. Kall, C.P. Jaroniec, Paramagnetic Ions Enable Tuning of Nuclear Relaxation Rates and Provide Long-Range Structural Restraints in Solid-State NMR of Proteins, J. Am. Chem. Soc. 131 (2009) 8108-8120.

[24] M.R. Ermácora, J.M. Delfino, B. Cuenoud, A. Schepartz, R.O. Fox, Conformationdependent cleavage of staphylococcal nuclease with a disulfide-linked iron chelate, Proc. Natl. Acad. Sci. 89 (1992) 6383-6387.

[25] X.-C. Su, G. Otting, Paramagnetic labelling of proteins and oligonucleotides for NMR, J. Biomol. NMR 46 (2010) 101-112.

[26] J. Heiliger, T. Matzel, E.C. Cetiner, H. Schwalbe, G. Kuenze, B. Corzilius, Sitespecific dynamic nuclear polarization in a Gd(iii)-labeled protein, Phys. Chem. Chem. Phys. 22 (2020) 25455-25466.

[27] S. Wang, R.A. Munro, S.Y. Kim, K.-H. Jung, L.S. Brown, V. Ladizhansky, Paramagnetic Relaxation Enhancement Reveals Oligomerization Interface of a Membrane Protein, J. Am. Chem. Soc. 134 (2012) 16995-16998.

[28] M. Tang, D.A. Berthold, C.M. Rienstra, Solid-State NMR of a Large Membrane Protein by Paramagnetic Relaxation Enhancement, J. Phys. Chem. Lett. 2 (2011) 1836-1841.

[29] E. Teissier, G. Zandomeneghi, A. Loquet, D. Lavillette, J.-P. Lavergne, R. Montserret, F.-L. Cosset, A. Böckmann, B.H. Meier, F. Penin, E.-I. Pécheur, Mechanism of Inhibition of Enveloped Virus Membrane Fusion by the Antiviral Drug Arbidol, PLoS ONE 6 (2011) e15874.

[30] V.Jirasko, A. Lends, N.-A. Lakomek, M.-L. Fogeron, M.E. Weber, A.A. Malär, S. Penzel, R. Bartenschlager, B.H. Meier, A. Böckmann, Dimer Organization of MembraneAssociated NS5A of Hepatitis C Virus as Determined by Highly Sensitive $1 \mathrm{H}-$ Detected Solid-State NMR, Angew. Chem. Int. Ed. 60 (2021) 5339-5347.

[31] X. Jia, H. Yagi, X.-C. Su, M. Stanton-Cook, T. Huber, G. Otting, Engineering [Ln (DPA)3]3 - binding sites in proteins: a widely applicable method for tagging proteins with lanthanide ions, J. Biomol. NMR 50 (2011) 411-420.

[32] Z. Wei, Y. Yang, Q.-F. Li, F. Huang, H.-H. Zuo, X.-C. Su, Noncovalent Tagging Proteins with Paramagnetic Lanthanide Complexes for Protein Study, Chem. Eur. J. 19 (2013) 5758-5764.

[33] R.M. Almeida, C.F.G.C. Geraldes, S.R. Pauleta, J.J.G. Moura, Gd(III) Chelates as NMR Probes of Protein-Protein Interactions. Case Study: Rubredoxin and Cytochrome c3, Inorg. Chem., 50 (2011) 10600-10607.

[34] J.-C. Hus, J.J. Prompers, R. Brüschweiler, Assignment Strategy for Proteins with Known Structure, J. Magn. Reson. 157 (2002) 119-123.

[35] M. Zweckstetter, Determination of molecular alignment tensors without backbone resonance assignment: Aid to rapid analysis of protein-protein interactions, J. Biomol. NMR 27 (2003) 41-56.

[36] G. Pintacuda, T. Huber, M. Keniry, A. Park, N. Dixon, G. Otting, Fast Assignments of 15N-HSQC Spectra of Proteins by Paramagnetic Labeling, in: G. Webb (Ed.), Modern Magnetic Resonance, Springer, Netherlands, 2006, pp. 1263-1269.

[37] P. Shealy, Y. Liu, M. Simin, H. Valafar, Backbone resonance assignment and order tensor estimation using residual dipolar couplings, J. Biomol. NMR 50 (2011) 357-369.

[38] C. Langmead, B. Donald, An expectation/maximization nuclear vector replacement algorithm for automated NMR resonance assignments, J. Biomol. NMR 29 (2004) 111-138.
[39] Y.-S. Jung, M. Zweckstetter, Backbone assignment of proteins with known structure using residual dipolar couplings, J. Biomol. NMR 30 (2004) 25-35.

[40] Y. Tian, C.D. Schwieters, S.J. Opella, F.M. Marassi, AssignFit: A program for simultaneous assignment and structure refinement from solid-state NMR spectra, J. Magn. Reson. 214 (2012) 42-50.

[41] T. Jovanovic, A.E. McDermott, Observation of Ligand Binding to Cytochrome P450 BM-3 by Means of Solid-State NMR Spectroscopy, J. Am. Chem. Soc. 127 (2005) 13816-13821.

[42] S. Balayssac, I. Bertini, A. Bhaumik, M. Lelli, C. Luchinat, Paramagnetic shifts in solid-state NMR of proteins to elicit structural information, Proc. Natl. Acad. Sci. 105 (2008) 17284-17289.

[43] J. Li, K.B. Pilla, Q. Li, Z. Zhang, X. Su, T. Huber, J. Yang, Magic Angle Spinning NMR Structure Determination of Proteins from Pseudocontact Shifts, J. Am. Chem. Soc. 135 (2013) 8294-8303.

[44] I. Bertini, A. Bhaumik, G. De Paëpe, R.G. Griffin, M. Lelli, J.R. Lewandowski, C. Luchinat, High-Resolution Solid-State NMR Structure of a 17.6 kDa Protein, J. Am. Chem. Soc. 132 (2009) 1032-1040.

[45] M.J. Knight, I.C. Felli, R. Pierattelli, I. Bertini, L. Emsley, T. Herrmann, G. Pintacuda, Rapid Measurement of Pseudocontact Shifts in Metalloproteins by Proton-Detected Solid-State NMR Spectroscopy, J. Am. Chem. Soc. 134 (2012) $14730-14733$.

[46] P.S. Nadaud, J.J. Helmus, N. Höfer, C.P. Jaroniec, Long-Range Structural Restraints in Spin-Labeled Proteins Probed by Solid-State Nuclear Magnetic Resonance Spectroscopy, J. Am. Chem. Soc. 129 (2007) 7502-7503.

[47] I. Sengupta, P.S. Nadaud, J.J. Helmus, C.D. Schwieters, C.P. Jaroniec, Protein fold determined by paramagnetic magic-angle spinning solid-state NMR spectroscopy, Nature Chem. 4 (2012) 410-417.

[48] I. Sengupta, P.S. Nadaud, C.P. Jaroniec, Protein Structure Determination with Paramagnetic Solid-State NMR Spectroscopy, Acc. Chem. Res. 46 (2013) 21172126.

[49] M. Stelter, I. Gutsche, U. Kapp, A. Bazin, G. Bajic, G. Goret, M. Jamin, J. Timmins, L. Terradot, Architecture of a Dodecameric Bacterial Replicative Helicase, Structure 20 (2012) 554.

[50] T. Wiegand, R. Cadalbert, D. Lacabanne, J. Timmins, L. Terradot, A. Bockmann, B.H. Meier, The conformational changes coupling ATP hydrolysis and translocation in a bacterial DnaB helicase, Nat. Commun. 10 (2019) 31.

[51] M. Spies, DNA Helicases and DNA Motor Proteins, Springer, New York, 2012.

[52] K. Takegoshi, S. Nakamura, T. Terao, 13C-1H dipolar-assisted rotational resonance in magic-angle spinning NMR, Chem. Phys. Lett. 344 (2001) 631637.

[53] K. Takegoshi, S. Nakamura, T. Terao, 13C-13C polarization transfer by resonant interference recoupling under magic-angle spinning in solid-state NMR, Chem. Phys. Lett. 307 (1999) 295-302.

[54] A. Bazin, M.V. Cherrier, I. Gutsche, J. Timmins, L. Terradot, Structure and primase-mediated activation of a bacterial dodecameric replicative helicase, Nucleic Acids Res. 43 (2015) 8564-8576.

[55] J.K. Leman, B.D. Weitzner, S.M. Lewis, J. Adolf-Bryfogle, N. Alam, R.F. Alford, M. Aprahamian, D. Baker, K.A. Barlow, P. Barth, B. Basanta, B.J. Bender, K. Blacklock, J. Bonet, S.E. Boyken, P. Bradley, C. Bystroff, P. Conway, S. Cooper, B. E. Correia, B. Coventry, R. Das, R.M. De Jong, F. DiMaio, L. Dsilva, R. Dunbrack, A. S. Ford, B. Frenz, D.Y. Fu, C. Geniesse, L. Goldschmidt, R. Gowthaman, J.J. Gray, D. Gront, S. Guffy, S. Horowitz, P.-S. Huang, T. Huber, T.M. Jacobs, J.R. Jeliazkov, D.K. Johnson, K. Kappel, J. Karanicolas, H. Khakzad, K.R. Khar, S.D. Khare, F. Khatib, A. Khramushin, I.C. King, R. Kleffner, B. Koepnick, T. Kortemme, G. Kuenze, B. Kuhlman, D. Kuroda, J.W. Labonte, J.K. Lai, G. Lapidoth, A. LeaverFay, S. Lindert, T. Linsky, N. London, J.H. Lubin, S. Lyskov, J. Maguire, L. Malmström, E. Marcos, O. Marcu, N.A. Marze, J. Meiler, R. Moretti, V.K. Mulligan, S. Nerli, C. Norn, S. Ó’Conchúir, N. Ollikainen, S. Ovchinnikov, M.S. Pacella, X. Pan, H. Park, R.E. Pavlovicz, M. Pethe, B.G. Pierce, K.B. Pilla, B. Raveh, P.D. Renfrew, S.S.R. Burman, A. Rubenstein, M.F. Sauer, A. Scheck, W. Schief, O. Schueler-Furman, Y. Sedan, A.M. Sevy, N.G. Sgourakis, L. Shi, J.B. Siegel, D.-A. Silva, S. Smith, Y. Song, A. Stein, M. Szegedy, F.D. Teets, S.B. Thyme, R.Y.-R. Wang, A. Watkins, L. Zimmerman, R. Bonneau, Macromolecular modeling and design in Rosetta: recent methods and frameworks, Nature Meth. 17 (2020) 665-680.

[56] D.N. Frick, C.C. Richardson, DNA Primases, Annu. Rev. Biochem 70 (2001) 3980.

[57] U.B. le Paige, S. Xiang, M.M.R.M. Hendrix, Y. Zhang, G.E. Folkers, M. Weingarth, A.M.J.J. Bonvin, T.G. Kutateladze, I.K. Voets, M. Baldus, H. van Ingen, Characterization of nucleosome sediments for protein interaction studies by solid-state NMR spectroscopy, Magn. Reson. 2 (2021) 187-202.

[58] C. Gardiennet, A.K. Schütz, A. Hunkeler, B. Kunert, L. Terradot, A. Böckmann, B.H Meier, A Sedimented Sample of a 59 kDa Dodecameric Helicase Yields HighResolution Solid-State NMR Spectra, Angew. Chem. Int. Ed. 51 (2012) 7855-7858.

[59] J. Iwahara, C.D. Schwieters, G.M. Clore, Ensemble Approach for NMR Structure Refinement against 1H Paramagnetic Relaxation Enhancement Data Arising from a Flexible Paramagnetic Group Attached to a Macromolecule, J. Am. Chem. Soc. 126 (2004) 5879-5896.

[60] S. Grazulis, D. Chateigner, R.T. Downs, A.F.T. Yokochi, M. Quiros, L. Lutterotti, E. Manakova, J. Butkus, P. Moeck, A. Le Bail, Crystallography Open Database - an open-access collection of crystal structures, J. Appl. Crystallogr. 42 (2009) $726-$ 729.

[61] S. Gražulis, A. Daškevič, A. Merkys, D. Chateigner, L. Lutterotti, M. Quirós, N.R. Serebryanaya, P. Moeck, R.T. Downs, A. Le Bail, Crystallography Open Database (COD): an open-access collection of crystal structures and platform for worldwide collaboration, Nucleic Acids Res. 40 (2011) D420-D427. 
[62] J. Koehler, J. Meiler, Expanding the utility of NMR restraints with paramagnetic compounds: Background and practical aspects, Prog. Nucl. Magn. Reson. Spectrosc. 59 (2011) 360-389.

[63] P. Rovó, K. Grohe, K. Giller, S. Becker, R. Linser, Proton Transverse Relaxation as a Sensitive Probe for Structure Determination in Solid Proteins, ChemPhysChem 16 (2015) 3791-3796.

[64] W. Li, Q. Zhang, J.J. Joos, P.F. Smet, J. Schmedt auf der Günne, Blind spheres of paramagnetic dopants in solid state NMR, Phys. Chem. Chem. Phys., 21 (2019) 10185-10194.

[65] I. Bertini, C. Luchinat, G. Parigi, E. Ravera, in: NMR of Paramagnetic Molecules, Second ed., Elsevier, Boston, 2017, pp. 1-24.

[66] G. Jeschke, Y. Polyhach, Distance measurements on spin-labelled biomacromolecules by pulsed electron paramagnetic resonance, Phys. Chem. Chem. Phys. 9 (2007) 1895-1910.

[67] O. Schiemann, T.F. Prisner, Long-range distance determinations in biomacromolecules by EPR spectroscopy, Quarterly Rev. Biophys. 40 (2007) $1-53$.

[68] Y.D. Tsvetkov, A.D. Milov, A.G. Maryasov, Russian Chem. Rev. 77 (2008) 487.

[69] D. Goldfarb, Gd3+ spin labeling for distance measurements by pulse EPR spectroscopy, Phys. Chem. Chem. Phys. 16 (2014) 9685-9699.

[70] T. Wiegand, C. Gardiennet, R. Cadalbert, D. Lacabanne, B. Kunert, L. Terradot, A. Böckmann, B.H. Meier, Variability and conservation of structural domains in divide-and-conquer approaches, J. Biomol. NMR 65 (2016) 79-86.

[71] I. Bertini, C. Luchinat, G. Parigi, E. Ravera, B. Reif, P. Turano, Solid-state NMR of proteins sedimented by ultracentrifugation, Proc. Natl. Acad. Sci. 108 (2011) $10396-10399$.

[72] T. Wiegand, D. Lacabanne, A. Torosyan, J. Boudet, R. Cadalbert, F.H. Allain, B.H. Meier, A. Bockmann, Sedimentation Yields Long-Term Stable Protein Samples as Shown by Solid-State NMR, Front. Mol. Biosci. 7 (2020) 17.

[73] A. Böckmann, C. Gardiennet, R. Verel, A. Hunkeler, A. Loquet, G. Pintacuda, L. Emsley, B. Meier, A. Lesage, Characterization of different water pools in solidstate NMR protein samples, J. Biomol. NMR 45 (2009) 319-327.

[74] P.L. Gor'kov, R. Witter, E.Y. Chekmenev, F. Nozirov, R. Fu, W.W. Brey, Low-E probe for 19F-1H NMR of dilute biological solids, J. Magn. Reson., 189 (2007) 182-189.

[75] R. Fogh, J. Ionides, E. Ulrich, W. Boucher, W. Vranken, J.P. Linge, M. Habeck, W. Rieping, T.N. Bhat, J. Westbrook, K. Henrick, G. Gilliland, H. Berman, J. Thornton, M. Nilges, J. Markley, E. Laue, The CCPN project: an interim report on a data model for the NMR community, Nat. Struct. Mol. Biol. 9 (2002) 416-418.

[76] T. Stevens, R. Fogh, W. Boucher, V. Higman, F. Eisenmenger, B. Bardiaux, B.-J. van Rossum, H. Oschkinat, E. Laue, A software framework for analysing solidstate MAS NMR data, J. Biomol. NMR 51 (2011) 437-447.
[77] W.F. Vranken, W. Boucher, T.J. Stevens, R.H. Fogh, A. Pajon, M. Llinas, E.L. Ulrich, J.L. Markley, J. Ionides, E.D. Laue, The CCPN data model for NMR spectroscopy: Development of a software pipeline, Proteins Struct. Funct. Bioinformat. 59 (2005) 687-696.

[78] M.D. Hanwell, D.E. Curtis, D.C. Lonie, T, Vandermeersch, E. Zurek, G.R. Hutchison, Avogadro: an advanced semantic chemical editor, visualization, and analysis platform, J. Cheminformat. 4 (2012) 17.

[79] M. Dolg, H. Stoll, A. Savin, H. Preuss, Energy-adjusted pseudopotentials for the rare earth elements, Theoret. Chim. Acta 75 (1989) 173-194.

[80] Y. Zhao, D.G. Truhlar, The M06 suite of density functionals for main group thermochemistry, thermochemical kinetics, noncovalent interactions, excited states, and transition elements: two new functionals and systematic testing of four M06-class functionals and 12 other functionals, Theor. Chem. Acc. 120 (2008) 215-241.

[81] J. Tomasi, B. Mennucci, R. Cammi, Quantum Mechanical Continuum Solvation Models, Chem. Rev. 105 (2005) 2999-3094.

[82] S. Kothiwale, J.L. Mendenhall, J. Meiler, BCL::Conf: small molecule conformational sampling using a knowledge based rotamer library, J. Cheminformat. 7 (2015) 47.

[83] R.F. Alford, A. Leaver-Fay, J.R. Jeliazkov, M.J. O’Meara, F.P. DiMaio, H. Park, M.V. Shapovalov, P.D. Renfrew, V.K. Mulligan, K. Kappel, J.W. Labonte, M.S. Pacella, R. Bonneau, P. Bradley, R.L. Dunbrack, R. Das, D. Baker, B. Kuhlman, T. Kortemme, J.J. Gray, The Rosetta All-Atom Energy Function for Macromolecular Modeling and Design, J. Chem. Theory Comput. 13 (2017) 3031-3048.

[84] D.T. Jones, Protein secondary structure prediction based on position-specific scoring matrices11Edited by G. Von Heijne, J. Mol. Biol. 292 (1999) 195-202.

[85] J.K. Leman, R. Mueller, M. Karakas, N. Woetzel, J. Meiler, Simultaneous prediction of protein secondary structure and transmembrane spans, Proteins Struct. Funct. Bioinformat. 81 (2013) 1127-1140.

[86] A.A. Canutescu, R.L. Dunbrack Jr., Cyclic coordinate descent: A robotics algorithm for protein loop closure, Protein Sci. 12 (2003) 963-972.

[87] Y. Song, F. DiMaio, Ray Y.-R. Wang, D. Kim, C. Miles, T.J. Brunette, J. Thompson, D. Baker, High-Resolution Comparative Modeling with RosettaCM, Structure, 21 (2013) 1735-1742.

[88] F. DiMaio, A. Leaver-Fay, P. Bradley, D. Baker, I. André, Modeling Symmetric Macromolecular Structures in Rosetta3, PLOS ONE 6 (2011) e20450.

[89] G. Hagelueken, R. Ward, J.H. Naismith, O. Schiemann, MtsslWizard. In Silico Spin-Labeling and Generation of Distance Distributions in PyMOL, Appl. Magn. Reson. 42 (2012) 377-391. 